\title{
Maximum scattered linear sets of pseudoregulus type and the Segre variety $\mathcal{S}_{n, n}$
}

\author{
G. Lunardon • G. Marino • O. Polverino • \\ R. Trombetti
}

Received: 24 September 2012 / Accepted: 5 August 2013 / Published online: 31 August 2013

(C) Springer Science+Business Media New York 2013

\begin{abstract}
In this paper we study a family of scattered $\mathbb{F}_{q}$-linear sets of rank $t n$ of the projective space $P G\left(2 n-1, q^{t}\right)(n \geq 1, t \geq 3)$, called of pseudoregulus type, generalizing results contained in Lavrauw and van de Voorde, Des. Codes Crypt. 20(1) (2013) and in Marino et al. J. Combin. Theory, Ser. A 114:769-788 (2007). As an application, we characterize, in terms of the associated linear sets, some classical families of semifields: the Generalized Twisted Fields and the 2-dimensional Knuth semifields.
\end{abstract}

Keywords Linear set $\cdot$ Subgeometry $\cdot$ Semifield

\section{Introduction}

In recent years the theory of linear sets has constantly increased its importance mainly because of its connection with other geometric objects such as blocking sets, translation ovoids and semifield planes (for an overview see [26]).

In this paper we study a family of maximum scattered $\mathbb{F}_{q}$-linear sets of the projective space $\Lambda=P G\left(2 n-1, q^{t}\right)(n \geq 1, t \geq 3)$. They were first introduced in [25] for

G. Lunardon · R. Trombetti

Dip. di Matematica e Applicazioni, Università di Napoli "Federico II", 80126 Napoli, Italy

G. Lunardon

e-mail: lunardon@unina.it

R. Trombetti

e-mail: rtrombet@unina.it

G. Marino $\cdot$ O. Polverino $(\bowtie)$

Dip. di Matematica e Fisica, Seconda Università degli Studi di Napoli, 81100 Caserta, Italy e-mail: olga.polverino@unina2.it

G. Marino

e-mail: giuseppe.marino@unina2.it 
$n=2$ and $t=3$, and further generalized in [18] for $n \geq 2$ and $t=3$. If $\Lambda$ is not a line, it is possible to associate with any such linear set a family of $\left(q^{n t}-1\right) /\left(q^{t}-1\right)$ pairwise disjoint lines admitting exactly two $(n-1)$-dimensional transversal spaces. Such a set of lines is called pseudoregulus, in analogy to the pseudoregulus of $P G\left(3, q^{2}\right)$ introduced by Freeman in [8]. For this reason, we refer to the relevant family of linear sets as linear sets of pseudoregulus type.

All maximum scattered $\mathbb{F}_{q}$-linear sets of $\Lambda=P G\left(2 n-1, q^{3}\right)(n \geq 2)$ are of pseudoregulus type and they are all equivalent under the action of the collineation group of $\Lambda$ (see [25, Propositions 2.7 and 2.8] for $n=2$ and [18, Sect. 3 and Theorem 4] for $n \geq 3)$. In this paper, we characterize $\mathbb{F}_{q}$-linear sets of $P G\left(2 n-1, q^{t}\right)(n \geq 1$, $t \geq 3$ ) of pseudoregulus type in terms of the associated projected subgeometry and we prove that, when $n>1$ there are $\varphi(t) / 2$ (where $\varphi$ denotes Euler's phi function) orbits of such $\mathbb{F}_{q}$-linear sets under the action of the collineation group of $P G\left(2 n-1, q^{t}\right)$ (Theorems 3.11, 3.13). Also, we show that, when $t \geq 4$ and $q>3$, there exist examples of maximum scattered $\mathbb{F}_{q}$-linear sets of $P G\left(2 n-1, q^{t}\right)(n \geq 1)$ which are not of pseudoregulus type (Example 4.6).

Finally, in Sect. 5 we first prove some geometric properties of the Segre Variety $\mathcal{S}_{n, n}$ of the projective space $\mathbb{P}=P G\left(n^{2}-1, q\right)$. These properties, together with the results contained in Sects. 3 and 4, allow us to describe and characterize the linear sets associated with some classical semifields: the Generalized Twisted Fields and the Knuth semifields 2-dimensional over their left nucleus (Propositions 5.5 and 5.9, Theorems 5.6 and 5.10).

\section{Preliminary results}

A $(t-1)$-spread of a projective space $P G(n t-1, q)$ is a family $\mathcal{S}$ of mutually disjoint subspaces of dimension $t-1$ such that each point of $P G(n t-1, q)$ belongs to an element of $\mathcal{S}$. A first example of spread can be obtained in the following way. Let $P G\left(n-1, q^{t}\right)=P G\left(V, \mathbb{F}_{q^{t}}\right)$. Any point $P$ of $P G\left(n-1, q^{t}\right)$ defines a $(t-1)$ dimensional subspace $X(P)$ of the projective space $P G(n t-1, q)=P G\left(V, \mathbb{F}_{q}\right)$ and $\mathcal{D}=\left\{X(P): P \in P G\left(n-1, q^{t}\right)\right\}$ is a spread of $P G(n t-1, q)$, called a Desarguesian spread (see [27], Sect. 25). ${ }^{1}$ If $n>2$, the incidence structure $\Pi_{n-1}(\mathcal{D})$, whose points are the elements of $\mathcal{D}$ and whose lines are the $(2 t-1)$-dimensional subspaces of $P G(n t-1, q)$ joining two distinct elements of $\mathcal{D}$, is isomorphic to $P G\left(n-1, q^{t}\right)$. The structure $\Pi_{n-1}(\mathcal{D})$ is called the $\mathbb{F}_{q}$-linear representation of $P G\left(n-1, q^{t}\right)$.

A Desarguesian $(t-1)$-spread of $P G(n t-1, q)$ can also be obtained as follows (see [27, Sect. 27], [20] and [3]). Embed $\Sigma \simeq P G(n t-1, q)$ in $\Sigma^{*}=P G\left(n t-1, q^{t}\right)$ in such a way that $\Sigma$ is the set of fixed points of a semilinear collineation $\Psi$ of $\Sigma^{*}$ of order $t$. Let $\Theta=P G\left(n-1, q^{t}\right)$ be a subspace of $\Sigma^{*}$ such that $\Theta, \Theta^{\Psi}, \ldots, \Theta^{\Psi^{t-1}}$ span the whole space $\Sigma^{*}$. If $P$ is a point of $\Theta, X^{*}(P)=\left\langle P, P^{\Psi}, \ldots, P^{\Psi^{t-1}}\right\rangle_{q}$ is a $(t-1)$-dimensional subspace of $\Sigma^{*}$ defining a $(t-1)$-dimensional subspace $X(P)=$ $X^{*}(P) \cap \Sigma$ of $\Sigma$. As $P$ varies over the subspace $\Theta$ we get a set of $q^{t(n-1)}+q^{t(n-2)}+$

\footnotetext{
${ }^{1}$ In [27] a Desarguesian spread is called "Sistema Grafico Elementare".
} 
$\cdots+q^{t}+1$ mutually disjoint $(t-1)$-dimensional subspaces of $\Sigma$. Such a set is denoted by $\mathcal{D}=\mathcal{D}(\Theta)$ and it turns out to be a Desarguesian $(t-1)$-spread of $\Sigma$. The $(n-1)$-dimensional subspaces $\Theta, \Theta^{\Psi}, \ldots, \Theta^{\Psi^{t-1}}$ are uniquely defined by the Desarguesian spread $\mathcal{D}$, i.e. $\mathcal{D}(\Theta)=\mathcal{D}(X)$ if and only if $X=\Theta^{\Psi^{i}}$ for some $i \in$ $\{0,1, \ldots, t-1\}$ and, following the terminology used by Segre [27, p. 29], we will refer to them as director spaces of $\mathcal{D}$ (see also [18, Theorem 3]).

Remark 2.1 Let $\mathcal{S}$ be a $(t-1)$-spread of $\Sigma=P G(n t-1, q)$ embedded in $\Sigma^{*}=$ $P G\left(n t-1, q^{t}\right)$ in such a way that $\Sigma=F i x(\Psi)$ where $\Psi$ is a semilinear collineation of $\Sigma^{*}$ of order $t$. If $H$ is an $(n-1)$-dimensional subspace of $\Sigma^{*}$ such that

(i) $\Sigma^{*}=\left\langle H, H^{\Psi}, \ldots, H^{\Psi^{t-1}}\right\rangle_{q^{t}}$;

(ii) $X^{*} \cap H \neq \emptyset$ for each $(t-1)$-dimensional subspace $X^{*}$ of $\Sigma^{*}$ such that $X^{*} \cap$ $\Sigma \in \mathcal{S}$

then it is easy to see that $\mathcal{D}(H)=\mathcal{S}$, i.e. $\mathcal{S}$ is a Desarguesian spread and $H$ is one of its director spaces.

\subsection{Linear sets}

Let $\Lambda=P G\left(r-1, q^{t}\right)=P G\left(V, \mathbb{F}_{q^{t}}\right), q=p^{h}, p$ prime, and let $L$ be a set of points of $\Lambda$. The set $L$ is said to be an $\mathbb{F}_{q}$-linear set of $\Lambda$ if it is defined by the non-zero vectors of an $\mathbb{F}_{q}$-vector subspace $U$ of $V$, i.e., $L=L_{U}=\left\{\langle\mathbf{u}\rangle_{q^{t}}: \mathbf{u} \in U \backslash\{\mathbf{0}\}\right\}$. If $\operatorname{dim}_{\mathbb{F}_{q}} U=k$, we say that $L$ has rank $k$. If $\Omega=P G\left(W, \mathbb{F}_{q^{t}}\right)$ is a subspace of $\Lambda$ and $L_{U}$ is an $\mathbb{F}_{q}$-linear set of $\Lambda$, then $\Omega \cap L_{U}$ is an $\mathbb{F}_{q}$-linear set of $\Omega$ defined by the $\mathbb{F}_{q}$ vector subspace $U \cap W$, and we say that $\Omega$ has weight $i$ in $L_{U}$ if $\operatorname{dim}_{\mathbb{F}_{q}}(W \cap U)=i$ and we write $\omega_{L_{U}}(\Omega)=i$. If $L_{U} \neq \emptyset$, we have

$$
\begin{aligned}
& \left|L_{U}\right| \leq q^{k-1}+q^{k-2}+\cdots+q+1, \\
& \left|L_{U}\right| \equiv 1(\bmod q) .
\end{aligned}
$$

For further details on linear sets see [26].

An $\mathbb{F}_{q}$-linear set $L_{U}$ of $\Lambda$ of rank $k$ is scattered if all of its points have weight 1 , or equivalently, if $L_{U}$ has maximum size $q^{k-1}+q^{k-2}+\cdots+q+1$.

In [4], the authors prove the following result on scattered linear sets.

Theorem 2.2 [4, Theorem 4.2] A scattered $\mathbb{F}_{q}$-linear set of $P G\left(r-1, q^{t}\right)$ has rank at most $r t / 2$.

A scattered $\mathbb{F}_{q}$-linear set $L$ of $P G\left(r-1, q^{t}\right)$ of maximum rank $r t / 2$ is called a maximum scattered linear set.

Remark 2.3 Note that if $L_{U}$ is a scattered $\mathbb{F}_{q}$-linear set of $P G\left(r-1, q^{t}\right)$ of rank $k$ containing more than one point, then by $\left|L_{U}\right|=q^{k-1}+q^{k-2}+\cdots+q+1$ and (2), $L_{U}$ is not an $\mathbb{F}_{q^{s}}$-linear set for each subfield $\mathbb{F}_{q^{s}}$ of $\mathbb{F}_{q^{t}}$ properly containing $\mathbb{F}_{q}$. In other words, a scattered $\mathbb{F}_{q}$-linear set $L$ of rank $k>1$ of $P G\left(r-1, q^{t}\right)$ is not a linear set of rank $n<k$. Also, by Theorem 2.2, a maximum scattered linear set of $P G\left(r-1, q^{t}\right)$ spans the whole space. 


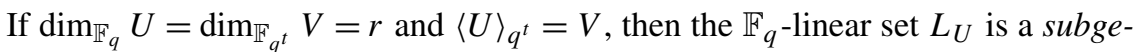
ometry of $P G\left(V, \mathbb{F}_{q^{t}}\right)=P G\left(r-1, q^{t}\right)$ isomorphic to $P G(r-1, q)$. If $t=2$, then $L_{U}$ is a Baer subgeometry of $P G\left(r-1, q^{2}\right)$.

In [23], the authors give the following characterization of $\mathbb{F}_{q}$-linear sets. Let $\Sigma=$ $P G(k-1, q)$ be a subgeometry of $\Sigma^{*}=P G\left(k-1, q^{t}\right)$, let $\Gamma$ be a $(k-r-1)$ dimensional subspace of $\Sigma^{*}$ disjoint from $\Sigma$ and let $\Lambda=P G\left(r-1, q^{t}\right)$ be an $(r-1)$ dimensional subspace of $\Sigma^{*}$ disjoint from $\Gamma$. Denote by

$$
L=\left\{\langle\Gamma, P\rangle_{q^{t}} \cap \Lambda: P \in \Sigma\right\}
$$

the projection of $\Sigma$ from $\Gamma$ to $\Lambda$. We call $\Gamma$ and $\Lambda$, respectively, the center and the axis of the projection. Denote by $p_{\Gamma, \Lambda}$ the map from $\Sigma$ to $L$ defined by $P \mapsto\langle\Gamma, P\rangle_{q^{t}} \cap \Lambda$ for each point $P$ of $\Sigma$. By definition $p_{\Gamma, \Lambda}$ is surjective and $L=p_{\Gamma, \Lambda}(\Sigma)$.

Theorem 2.4 [23, Theorems 1 and 2] If $L$ is a projection of $\Sigma=P G(k-1, q)$ to $\Lambda=$ $P G\left(r-1, q^{t}\right)$, then $L$ is an $\mathbb{F}_{q}$-linear set of $\Lambda$ of rank $k$ and $\langle L\rangle_{q^{t}}=\Lambda$. Conversely, if $L$ is an $\mathbb{F}_{q}$-linear set of $\Lambda$ of rank $k$ and $\langle L\rangle_{q^{t}}=\Lambda$, then either $L$ is a subgeometry of $\Lambda$ or for each $(k-r-1)$-dimensional subspace $\Gamma$ of $\Sigma^{*}=P G\left(k-1, q^{t}\right)$ disjoint from $\Lambda$ there exists a subgeometry $\Sigma$ of $\Sigma^{*}$ disjoint from $\Gamma$ such that $L=p_{\Gamma, \Lambda}(\Sigma)$.

Also, in [17] it has been proven:

Theorem 2.5 [17, Theorem 3] Let $L_{1}=p_{\Gamma_{1}, \Lambda}\left(\Sigma_{1}\right)$ and $L_{2}=p_{\Gamma_{2}, \Lambda}\left(\Sigma_{2}\right)$ be two $\mathbb{F}_{q}$ linear sets of rank $k$ of $\Lambda=\left\langle L_{1}\right\rangle_{q^{t}}=\left\langle L_{2}\right\rangle_{q^{t}}$, and suppose that $L_{i}$ is not a linear set of rank $n<k$. Then $L_{1}$ and $L_{2}$ are projectively equivalent if and only if there exists $\beta \in \operatorname{Aut}\left(\Sigma^{*}\right)$ such that $\Sigma_{1}^{\beta}=\Sigma_{2}$ and $\Gamma_{1}^{\beta}=\Gamma_{2}$.

Remark 2.6 Note that, if $S_{\Gamma}=\Sigma^{*} / \Gamma \simeq P G\left(r-1, q^{t}\right)$ denotes the $(r-1)$ dimensional space obtained as quotient geometry of $\Sigma^{*}$ over $\Gamma$, then the set $L_{\Gamma, \Sigma}$ of the $(k-r)$-dimensional subspaces of $\Sigma^{*}$ containing $\Gamma$ and with non-empty intersection with $\Sigma$ is an $\mathbb{F}_{q}$-linear set of the space $S_{\Gamma}$ isomorphic to $L=p_{\Gamma, \Lambda}(\Sigma)$, for each $(r-1)$-dimensional space $\Lambda$ disjoint from $\Gamma$. This means that $p_{\Gamma, \Lambda}(\Sigma)$ is isomorphic to the $\mathbb{F}_{q}$-linear set $\{P+\Gamma: P \in \Sigma\}$ of the quotient space $S_{\Gamma}$, and hence it does not depend on the choice of the axis $\Lambda$, and we will simply denote it as $p_{\Gamma}(\Sigma)$.

\section{Maximum scattered $\mathbb{F}_{q}$-linear sets of pseudoregulus type in $\operatorname{PG}\left(2 n-1, q^{t}\right)$}

In this section we study a family of maximum scattered linear sets to which a geometric structure, called pseudoregulus, can be associated. This generalizes results contained in $[15,25]$ and [18].

Definition 3.1 Let $L=L_{U}$ be a scattered $\mathbb{F}_{q}$-linear set of $\Lambda=P G\left(2 n-1, q^{t}\right)$ of rank $t n, t, n \geq 2$. We say that $L$ is of pseudoregulus type if 
(i) there exist $m=\frac{q^{n t}-1}{q^{t}-1}$ pairwise disjoint lines of $\Lambda$, say $s_{1}, s_{2}, \ldots, s_{m}$, such that

$$
w_{L}\left(s_{i}\right)=t, \quad \text { i.e. }\left|L \cap s_{i}\right|=q^{t-1}+q^{t-2}+\cdots+q+1 \forall i=1, \ldots, m ;
$$

(ii) there exist exactly two $(n-1)$-dimensional subspaces $T_{1}$ and $T_{2}$ of $\Lambda$ disjoint from $L$ such that $T_{j} \cap s_{i} \neq \emptyset$ for each $i=1, \ldots, m$ and $j=1,2$.

We call the set of lines $\mathcal{P}_{L}=\left\{s_{i}: i=1, \ldots, m\right\}$ the $\mathbb{F}_{q}$-pseudoregulus (or simply pseudoregulus) of $\Lambda$ associated with $L$ and we refer to $T_{1}$ and $T_{2}$ as transversal spaces of $\mathcal{P}_{L}$ (or transversal spaces of $L$ ). Note that, by Remark 2.3, $L$ spans the whole space and hence the transversal spaces $T_{1}$ and $T_{2}$ are disjoint. When $t=n=2$, these objects already appeared in [8], where the term "pseudoregulus" was introduced for the first time.

If $L$ is a scattered $\mathbb{F}_{q}$-linear set of the projective space $P G\left(r-1, q^{t}\right)$, then by Theorem 2.2 every $h$-dimensional subspace $X_{h}$ of $P G\left(r-1, q^{t}\right)$ intersects $L$ in a linear set of rank at most $(h+1) t / 2$, i.e. the weight of $X_{h}$ in $L$ is at most $(h+1) t / 2$. So we get:

Proposition 3.2 If $t \geq 3$, then the lines of weight $t$ in a scattered $\mathbb{F}_{q}$-linear set $L$ of $P G\left(r-1, q^{t}\right)$ of rank $h$ are pairwise disjoint and hence the number of such lines is at most $\left(q^{h}-1\right) /\left(q^{t}-1\right)$.

Proof If $\ell$ and $\ell^{\prime}$ are distinct lines of $P G\left(r-1, q^{t}\right)$ of weight $t$ in $L$ and $\ell \cap \ell^{\prime} \neq \emptyset$, then the plane $\pi$ joining $\ell$ and $\ell^{\prime}$ has weight at least $2 t-1$ in $L$. On the other hand, since $\pi \cap L$ is a scattered $\mathbb{F}_{q}$-linear set of the plane $\pi$, by Theorem 2.2, we also have that the weight of $\pi$ in $L$ is at most $3 t / 2$; so we get $t \leq 2$, a contradiction. Hence, the number of lines of $P G\left(r-1, q^{t}\right)$ having weight $t$ in $L$ is at most

$$
\frac{q^{h-1}+q^{h-2}+\cdots+q+1}{q^{t-1}+q^{t-2}+\cdots+q+1}=\frac{q^{h}-1}{q^{t}-1} .
$$

As a consequence of Proposition 3.2 we get:

Corollary 3.3 If $L$ is an $\mathbb{F}_{q}$-linear set of pseudoregulus type of the projective space $\Lambda=P G\left(2 n-1, q^{t}\right)$, with $t \geq 3$, then the associated pseudoregulus is the set of all the lines of $\Lambda$ of weight $t$ in L. Hence, the pseudoregulus associated with $L$ and its transversal spaces are uniquely determined.

Remark 3.4 If $t=2$, a scattered $\mathbb{F}_{q}$-linear set $L$ of rank $2 n$ of the projective space $\Lambda$ is a Baer subgeometry isomorphic to $\operatorname{PG}(2 n-1, q)$ and each line spread of $L$ produces a set of lines of $\Lambda$ satisfying (i) of Definition 3.1 and each Desarguesian line spread, say $\mathcal{D}$, of $L$ gives a set of lines of $\Lambda$ satisfying both (i) and (ii) of Definition 3.1. In this last case the transversal spaces are the two $(n-1)$-dimensional director spaces of the Desarguesian spread $\mathcal{D}$. So each maximum scattered $\mathbb{F}_{q}$-linear 
set $L$ of the projective space $\Lambda=P G\left(2 n-1, q^{2}\right)$ is of pseudoregulus type, but in this case the associated pseudoregulus is not uniquely defined; also, since these linear sets are Baer subgeometries, they are all projectively equivalent. The same happens in the next case, i.e. each maximum scattered $\mathbb{F}_{q}$-linear set $L$ of the projective space $\Lambda=P G\left(2 n-1, q^{3}\right)(n \geq 2)$ is of pseudoregulus type and they are all projectively equivalent (see [25] and [18, Theorem 4, Lemmas 5, 7, Theorem 10]). Whereas, when $t>3$ : (i) there exist maximum scattered $\mathbb{F}_{q}$-linear sets of the projective space $\Lambda=P G\left(2 n-1, q^{t}\right)$ which are not of pseudoregulus type (see Example 4.6); (ii) $\mathbb{F}_{q^{-}}$ linear sets of pseudoregulus type, in general, are not all projectively equivalent (see Theorem 3.13).

The construction presented in [15, Sect. 2] when $t=3$ and $n=2$, can be generalized providing a simple way to construct scattered $\mathbb{F}_{q}$-linear sets of pseudoregulus type of $P G\left(2 n-1, q^{t}\right)$ for any $t, n \geq 2$.

Let $\Lambda=P G\left(V, \mathbb{F}_{q^{t}}\right)$, where $V=V\left(2 n, \mathbb{F}_{q^{t}}\right)=U_{1} \oplus U_{2}$, with $\operatorname{dim} U_{1}=\operatorname{dim} U_{2}=$ $n$ and let $T_{1}=P G\left(U_{1}, \mathbb{F}_{q^{t}}\right)$ and $T_{2}=P G\left(U_{2}, \mathbb{F}_{q^{t}}\right)$. Now, let $\Phi_{f}$ be a semilinear collineation between $T_{1}$ and $T_{2}$, induced by the invertible semilinear map $f: U_{1} \rightarrow$ $U_{2}$, having as companion automorphism an element $\sigma \in \operatorname{Aut}\left(\mathbb{F}_{q^{t}}\right)$ such that $\operatorname{Fix}(\sigma)=$ $\mathbb{F}_{q}$. Then, for each $\rho \in \mathbb{F}_{q^{t}}^{*}$, the set

$$
W_{\rho, f}=\left\{\underline{u}+\rho f(\underline{u}): \underline{u} \in U_{1}\right\}
$$

is an $\mathbb{F}_{q}$-vector subspace of $V$ of dimension $t n$ and it is not difficult to see that $L=$ $L_{W_{\rho, f}}$ is an $\mathbb{F}_{q}$-linear set of $\Lambda$ of rank $t n$ of scattered type. ${ }^{2}$ Also, we can see that for each line $s_{P}$ joining the points $P=\langle\underline{u}\rangle_{q^{t}}$ and $P^{\Phi_{f}}=\langle f(\underline{u})\rangle_{q^{t}}$ of $T_{1}$ and $T_{2}$ respectively, we have that

$$
s_{P} \cap L=\left\{\left\langle\lambda \underline{u}+\lambda^{\sigma} f(\underline{u})\right\rangle_{q^{t}}: \lambda \in \mathbb{F}_{q^{t}}^{*}\right\} .
$$

Hence, the line $s_{P}$, for each $P \in T_{1}$, has weight $t$ in $L$. Also, if $P \neq Q$, the lines $s_{P}$ and $s_{Q}$ are disjoint. This means that $L$ satisfies (i) of Definition 3.1. Moreover, it is clear that $T_{1} \cap s_{P}=\{P\}$ and $T_{2} \cap s_{P}=\left\{P^{\Phi_{f}}\right\}$ for each $P \in T_{1}$ and that $T_{1} \cap L=$ $T_{2} \cap L=\emptyset$. In addition, $T_{1}$ and $T_{2}$ are the only $(n-1)$-dimensional transversal spaces of the lines $s_{P}$. Indeed, if $T=P G\left(U, \mathbb{F}_{q^{t}}\right)=P G\left(n-1, q^{t}\right)$ were another transversal space, then $T$ would be disjoint from $T_{1}$ and $T_{2}$ and, since $T \cap s_{P} \neq \emptyset$ for each $P \in T_{1}$, we have that

$$
U=\left\{\underline{u}+\lambda_{\underline{u}} f(\underline{u}): \underline{u} \in U_{1}\right\},
$$

where $\lambda_{\underline{u}} \in \mathbb{F}_{q^{t}}$ for each $\underline{u} \in U_{1}$ and $\lambda_{\underline{u}} \neq 0$ for each $\underline{u} \neq \underline{0}$. Now, since $U$ is an $\mathbb{F}_{q^{t}}$ subspace of $V$, the map $f$ turns out to be an $\mathbb{F}_{q^{t}}$-linear map of $V$, a contradiction. So, also $L$ satisfies (ii) of Definition 3.1 and hence $L_{W_{\rho, f}}$ is a maximum scattered $\mathbb{F}_{q}$-linear set of $\Lambda$ of pseudoregulus type and $\mathcal{P}_{L}=\left\{s_{P}: P \in T_{1}\right\}$ is its associated pseudoregulus. Hence we have proved the following:

\footnotetext{
${ }^{2}$ More generally, if $\operatorname{Fix}(\sigma)=\mathbb{F}_{q^{\prime}}$, then $L_{W_{\rho, f}}$ is an $\mathbb{F}_{q^{\prime}}$-linear set of $\Lambda$ of scattered type.
} 
Theorem 3.5 Let $T_{1}=P G\left(U_{1}, \mathbb{F}_{q^{t}}\right)$ and $T_{2}=P G\left(U_{2}, \mathbb{F}_{q^{t}}\right)$ be two disjoint $(n-1)$ dimensional subspaces of $\Lambda=P G\left(V, \mathbb{F}_{q^{t}}\right)=P G\left(2 n-1, q^{t}\right)(t>1)$ and let $\Phi_{f}$ be a semilinear collineation between $T_{1}$ and $T_{2}$ having as companion automorphism an element $\sigma \in \operatorname{Aut}\left(\mathbb{F}_{q^{t}}\right)$ such that Fix $(\sigma)=\mathbb{F}_{q}$. Then, for each $\rho \in \mathbb{F}_{q^{t}}^{*}$, the set

$$
L_{\rho, f}=\left\{\langle\underline{u}+\rho f(\underline{u})\rangle_{q^{t}}: \underline{u} \in U_{1} \backslash\{\underline{0}\}\right\}
$$

is an $\mathbb{F}_{q}$-linear set of $\Lambda$ of pseudoregulus type whose associated pseudoregulus is $\mathcal{P}_{L_{\rho, f}}=\left\{\left\langle P, P^{\Phi_{f}}\right\rangle_{q^{t}}: P \in T_{1}\right\}$, with transversal spaces $T_{1}$ and $T_{2}$.

Remark 3.6 Note that, with the notation of the previous theorem, if $L_{\rho, f} \cap L_{\rho^{\prime}, f} \neq \emptyset$, then $L_{\rho, f}=L_{\rho^{\prime}, f}$ and this happens if and only if $N_{q^{t} / q}(\rho)=N_{q^{t} / q}\left(\rho^{\prime}\right) .{ }^{3}$ Hence $T_{1}, T_{2}$ and the collineation $\Phi_{f}$ define a set of $q-1$ mutually disjoint linear sets of pseudoregulus type admitting the same associated pseudoregulus $\mathcal{P}$ and covering, together with the transversal spaces $T_{1}$ and $T_{2}$, the point set of $\mathcal{P}$.

Up to projective equivalence, the scattered $\mathbb{F}_{q}$-linear sets $L_{\rho, f}$ only depend on the field automorphism associated with $f$. Indeed, we have:

Theorem 3.7 The $\mathbb{F}_{q}$-linear sets of $\Lambda=P G\left(2 n-1, q^{t}\right)(n \geq 2, t \geq 2) L_{\rho, f}$ and $L_{\rho^{\prime}, g}$ are $P \Gamma L$-equivalent if and only if $\sigma_{f}=\sigma_{g}^{ \pm 1}$ where $\sigma_{f}$ and $\sigma_{g}$ are the automorphisms associated with $f$ and $g$, respectively.

Proof If $t=2$, the assertion follows from Remark 3.4. Let $t>2$. Then, by Corollary 3.3 the transversal spaces associated with $L_{\rho, f}$ and $L_{\rho^{\prime}, g}$ are uniquely determined. Hence, up to the action of $\operatorname{PGL}\left(2 n, q^{t}\right)$, we may assume that the transversal spaces of $L_{\rho, f}$ and $L_{\rho^{\prime}, g}$ are the same. Also, since $L_{\rho, f}=L_{1, \rho^{-1} f}$, we may consider scattered $\mathbb{F}_{q}$-linear sets of the form $L_{1, f}$. Suppose that $L_{1, f}$ and $L_{1, g}$ are projectively equivalent; i.e., there exists a collineation $\phi_{F}$ of $\Lambda=P G\left(2 n-1, q^{t}\right)=P G\left(V, \mathbb{F}_{q^{t}}\right)$ defined by an invertible semilinear map $F$ of the vector space $V$ having companion automorphism $\tau$, such that $\phi_{F}\left(L_{1, f}\right)=L_{1, g}$. By Corollary $3.3 \phi_{F}\left(\left\{T_{1}, T_{2}\right\}\right)=$ $\left\{T_{1}, T_{2}\right\}$. Precisely, either $\phi_{F}\left(T_{i}\right)=T_{i}, i=1,2$ or $\phi_{F}\left(T_{i}\right)=T_{j},\{i, j\}=\{1,2\}$.

In the first case, we have that $F\left(U_{1}\right)=U_{1}$ and $F\left(U_{2}\right)=U_{2}$. Since $\phi_{F}\left(L_{1, f}\right)=$ $L_{1, g}$, for each $\underline{u} \in U_{1}$ we have $\phi_{F}\left(\langle\underline{u}+f(\underline{u})\rangle_{q^{t}}\right)=\langle F(\underline{u}+f(\underline{u}))\rangle_{q^{t}} \in L_{1, g}$; in other words, for each vector $\underline{u} \in U_{1}$ we have

$$
F(\underline{u}+f(\underline{u}))=\lambda_{\underline{u}}\left(\underline{u}^{\prime}+g\left(\underline{u}^{\prime}\right)\right)=\lambda_{\underline{u}} \underline{u}^{\prime}+\lambda_{\underline{u}} g\left(\underline{u}^{\prime}\right),
$$

where $\underline{u}^{\prime} \in U_{1}$ and $\lambda_{\underline{u}} \in \mathbb{F}_{q^{t}}^{*}$ if $\underline{u} \neq \underline{0}$. On the other hand, we also have $F(\underline{u}+f(\underline{u}))=$ $F(\underline{u})+F(f(\underline{u}))$, with $F(\underline{u}) \in U_{1}$ and $F(f(\underline{u})) \in U_{2}$. Taking this fact into account, since $V=U_{1} \oplus U_{2}$, Eq. (4) implies that $F(\underline{u})=\lambda_{\underline{u}} \underline{u}^{\prime}$ and $F(f(\underline{u}))=\lambda_{\underline{u}} g\left(\underline{u}^{\prime}\right)$. Hence

$$
F(f(\underline{u}))=\lambda_{\underline{u}} g\left(\frac{F(\underline{u})}{\lambda_{\underline{u}}}\right)=\frac{\lambda_{\underline{u}}}{\lambda_{\underline{u}}^{\sigma_{g}}} g(F(\underline{u})) .
$$

\footnotetext{
${ }^{3}$ Here $N_{q^{t} / q}(\cdot)$ denotes the norm function from $\mathbb{F}_{q^{t}}$ on $\mathbb{F}_{q}$.
} 
Let now $\underline{u}$ and $\underline{v}$ be two non-zero vectors of $U_{1}$. If $\underline{u}$ and $\underline{v}$ are $\mathbb{F}_{q^{t}}$-independent, by Eq. (5) we have

$$
F(f(\underline{u}+\underline{v}))=\lambda_{\underline{u}+\underline{v}}^{1-\sigma_{g}} g(F(\underline{u}))+\lambda_{\underline{u}+\underline{v}}^{1-\sigma_{g}} g(F(\underline{v})) .
$$

Also, we get

$$
F(f(\underline{u}+\underline{v}))=F(f(\underline{u}))+F(f(\underline{v}))=\lambda_{\underline{u}}^{1-\sigma_{g}} g(F(\underline{u}))+\lambda_{\underline{v}}^{1-\sigma_{g}} g(F(\underline{v})) .
$$

Hence $\lambda_{\underline{u}}^{1-\sigma_{g}}=\lambda_{\underline{u}+\underline{v}}^{1-\sigma_{g}}=\lambda_{\underline{v}}^{1-\sigma_{g}}$, which implies $\lambda_{\underline{u}} / \lambda_{\underline{v}} \in \mathbb{F}_{q}$ since $\operatorname{Fix}\left(\sigma_{g}\right)=\mathbb{F}_{q}$.

On the other hand, if $\underline{u}$ and $\underline{v}$ are $\mathbb{F}_{q^{t}}$-dependent, choosing a vector $\underline{w} \in U_{1}$, such that $\underline{w} \notin\langle\underline{u}\rangle_{q^{t}}$, and arguing as above we get $\lambda_{\underline{u}} / \lambda_{\underline{v}} \in \mathbb{F}_{q}$.

This means that for each $\underline{u}, \underline{v} \in U_{1}$ there exists an element $\beta_{\underline{u}, \underline{v}} \in \mathbb{F}_{q}$ such that $\lambda_{\underline{u}}=\beta_{\underline{u}, \underline{v}} \lambda_{\underline{v}}$.

Let $\underline{u} \in \bar{U}_{1}, \underline{u} \neq \underline{0}$. Then, by Eq. (5), we get

$$
F(f(\alpha \underline{u}))=\alpha^{\sigma_{f} \tau} \lambda_{\underline{u}}^{1-\sigma_{g}} g(F(\underline{u}))
$$

for each $\alpha \in \mathbb{F}_{q^{t}}$. On the other hand, again by Eq. (5), for each $\alpha \in \mathbb{F}_{q^{t}}$ we have

$$
F(f(\alpha \underline{u}))=\lambda_{\alpha \underline{u}}^{1-\sigma_{g}} \alpha^{\tau \sigma_{g}} g(F(\underline{u})),
$$

where $\lambda_{\alpha u} \in \mathbb{F}_{q^{t}}$. Since $\lambda_{u} / \lambda_{\alpha u} \in \mathbb{F}_{q}$, we have $\lambda_{\alpha u}^{1-\sigma_{g}}=\lambda_{u}^{1-\sigma_{g}}$. Taking into account this fact, by Eqs. (6) and (7) we get $\alpha^{\sigma_{f} \tau}=\alpha^{\tau \sigma_{g}}$ for each $\alpha \in \mathbb{F}_{q^{t}}$, which implies $\sigma_{f}=\sigma_{g}$.

In the second case we have $F\left(U_{1}\right)=U_{2}$ and $F\left(U_{2}\right)=U_{1}$ and arguing as in the previous case we get $\sigma_{g}=\sigma_{f}^{-1}$.

Conversely, suppose that $\sigma_{f}=\sigma_{g}$ and let $\phi_{F}$ be the collineation of $\Lambda$ defined by the map $F$ of the vector space $V=U_{1} \oplus U_{2}$ defined as follows:

$$
F\left(\underline{u}_{1}+\underline{u}_{2}\right)=\underline{u}_{1}+g\left(f^{-1}\left(\underline{u}_{2}\right)\right),
$$

where $\underline{u}_{1} \in U_{1}$ and $\underline{u}_{2} \in U_{2}$. Then $\phi_{F}\left(L_{1, f}\right)=L_{1, g}$. On the other hand, if $\sigma_{f}=\sigma_{g}^{-1}$, the collineation $\phi_{F}$ of $\Lambda$ defined by the following map $F$ of $V=U_{1} \oplus U_{2}$

$$
F\left(\underline{u}_{1}+\underline{u}_{2}\right)=g\left(\underline{u}_{1}\right)+f^{-1}\left(\underline{u}_{2}\right)
$$

sends $L_{1, f}$ to $L_{1, g}$. This concludes the proof.

As a consequence of Theorem 3.7 we have the following:

Corollary 3.8 In the projective space $\Lambda=P G\left(2 n-1, q^{t}\right)(n \geq 2, t \geq 3)$ there are $\varphi(t) / 2$ orbits of scattered $\mathbb{F}_{q}$-linear sets of $\Lambda$ of rank tn of type $L_{\rho, f}$ under the action of the collineation group of $\Lambda$.

Proof By the previous theorem, two linear sets $L_{\rho, f}$ and $L_{\rho, g}$ are $P \Gamma L$-equivalent if and only if either $\sigma_{f}=\sigma_{g}$ or $\sigma_{f}=\sigma_{g}^{-1}$. So the number of orbits of such $\mathbb{F}_{q}$-linear sets 
under the action of $P \Gamma L\left(2 n, q^{t}\right)$ is $\chi / 2$ where $\chi$ is the number of $\mathbb{F}_{q}$-automorphisms $\sigma$ of $\mathbb{F}_{q^{t}}$ with $\operatorname{Fix}(\sigma)=\mathbb{F}_{q}$. This means that $\chi$ is the number of generators of the group $\operatorname{Gal}\left(\mathbb{F}_{q^{t}}: \mathbb{F}_{q}\right)$, i.e. $\chi=\varphi(t)$ is the number of positive integers less than $t$ and coprime with $t$.

In what follows we will show that each scattered $\mathbb{F}_{q}$-linear set of pseudoregulus type can be obtained as in Theorem 3.5. Let us start by proving the following:

Theorem 3.9 Let $\Sigma \simeq P G(t n-1, q)$ be a subgeometry of $\Sigma^{*}=P G\left(V, \mathbb{F}_{q^{t}}\right)=$ $P G\left(t n-1, q^{t}\right)$ defined by the semilinear collineation $\Psi$ of order $t$ of $\Sigma^{*}$. Also, let $\mathcal{D}$ be a Desarguesian $(t-1)$-spread of $\Sigma$ and denote by $\Theta$ a director subspace of $\mathcal{D}$. Then, for each pair of integers $i_{1}, i_{2} \in\{0,1, \ldots, t-1\}$ such that $\operatorname{gcd}\left(i_{2}-i_{1}, t\right)=1$, the linear set obtained by projecting $\Sigma$ from the subspace $\Gamma=\left\langle\Theta^{\Psi^{i}}: i \neq i_{1}, i_{2}\right\rangle_{q^{t}}$ to $\Lambda=\left\langle\Theta^{\Psi^{i_{1}}}, \Theta^{\Psi^{i_{2}}}\right\rangle_{q^{t}}$ is a scattered $\mathbb{F}_{q}$-linear set of type $L_{\rho, f}$ described in Theorem 3.5.

Proof Since $\operatorname{Fix}(\Psi)=\Sigma$, the collineation $\Psi$ is induced by an invertible semilinear map $g: V \longrightarrow V$ of order $t>1$, with companion automorphism $\sigma$ such that Fix $(\sigma)=\mathbb{F}_{q}$. Since $\Theta=P G\left(U, \mathbb{F}_{q^{t}}\right)$ is a director subspace of the Desarguesian spread $\mathcal{D}$, we have that $\mathcal{D}=\mathcal{D}(\Theta)$ and $\Sigma^{*}=\left\langle\Theta, \Theta^{\Psi}, \ldots, \Theta^{\Psi^{t-1}}\right\rangle_{q^{t}}$. Let $i_{1}, i_{2} \in$ $\{0,1, \ldots, t-1\}$ be such that $\operatorname{gcd}\left(i_{2}-i_{1}, t\right)=1$ and let $\Gamma=\left\langle\Theta^{\Psi^{i}}: i \neq i_{1}, i_{2}\right\rangle_{q^{t}}$ and $\Lambda=\left\langle\Theta^{\Psi^{i_{1}}}, \Theta^{\Psi^{i_{2}}}\right\rangle_{q^{t}}$. Then $\operatorname{dim} \Gamma=n(t-2)-1, \operatorname{dim} \Lambda=2 n-1, \Gamma \cap \Lambda=$ $\Gamma \cap \Sigma=\emptyset$ and hence we can project the subgeometry $\Sigma$ from the center $\Gamma$ to the axis $\Lambda$. By Theorem 2.4, the projection $L=p_{\Gamma}(\Sigma)$ is an $\mathbb{F}_{q}$-linear set of $\Lambda$ of rank tn and $\langle L\rangle_{q^{t}}=\Lambda$. Also, it is easy to see that

$$
\begin{aligned}
\Sigma & =F i x(\Psi) \\
& =\left\{\left\langle\underline{u}+g(\underline{u})+g^{2}(\underline{u})+\cdots+\left.g^{t-1}(\underline{u})\right|_{q^{t}}: \underline{u} \in U \backslash\{\underline{0}\}\right\},\right.
\end{aligned}
$$

and hence the projection of $\Sigma$ from $\Gamma$ into $\Lambda$ is

$$
L=p_{\Gamma}(\Sigma)=\left\{\left\langle g^{i_{1}}(\underline{u})+g^{i_{2}}(\underline{u})\right\rangle_{q^{t}}: \underline{u} \in U \backslash\{\underline{0}\}\right\}=\left\{\left\langle\underline{v}+\left.f(\underline{v})\right|_{q^{t}}: \underline{v} \in g^{i_{1}}(U) \backslash\{\underline{0}\}\right\},\right.
$$

where $f: \underline{v} \in g^{i_{1}}(U) \mapsto g^{i_{2}-i_{1}}(\underline{v}) \in g^{i_{2}}(U)$. Since $f$ is an invertible semilinear map whose companion automorphism is $\sigma^{i_{2}-i_{1}}$ and $\operatorname{gcd}\left(i_{2}-i_{1}, t\right)=1$, we have that $\operatorname{Fix}\left(\sigma^{i_{2}-i_{1}}\right)=\mathbb{F}_{q}$. So, by Theorem 3.5, $L$ is a scattered $\mathbb{F}_{q}$-linear set of $\Lambda$ of pseudoregulus type with $\Theta^{\Psi^{i_{1}}}$ and $\Theta^{\Psi^{i_{2}}}$ as transversal spaces.

Remark 3.10 Note that, if $\operatorname{gcd}\left(i_{2}-i_{1}, t\right)=s$, in the previous proof we have $F i x\left(\sigma^{i_{2}-i_{1}}\right)=\mathbb{F}_{q^{s}}$, and hence the linear set $L$ obtained projecting $\Sigma$ from $\Gamma=\left\langle\Theta^{\Psi^{i}}\right.$ : $\left.i \neq i_{1}, i_{2}\right\rangle_{q^{t}}$, is an $\mathbb{F}_{q^{s}}$-linear set.

Recall that, by Theorem 2.4, every $\mathbb{F}_{q}$-linear set $L$ of $\Lambda$ spanning the whole space can be obtained projecting a suitable subgeometry. If $L$ is of pseudoregulus type we can prove the following: 
Theorem 3.11 Put $\Lambda=P G\left(2 n-1, q^{t}\right), \Sigma^{*}=P G\left(t n-1, q^{t}\right)$ and $\Sigma=F i x(\Psi) \simeq$ $P G(t n-1, q)$ where $\Psi$ is a semilinear collineation of $\Sigma^{*}$ of order $t$. Let $L$ be a scattered $\mathbb{F}_{q}$-linear set of $\Lambda$ of pseudoregulus type with associated pseudoregulus $\mathcal{P}$ obtained by projecting $\Sigma$ into $\Lambda$ from an $(n(t-2)-1)$-dimensional subspace $\Gamma$ disjoint from $\Sigma$. Then

(i) the set

$$
\mathcal{D}_{L}=\left\{\langle\Gamma, s\rangle_{q^{t}} \cap \Sigma: s \in \mathcal{P}\right\}
$$

is a Desarguesian $(t-1)$-spread of $\Sigma$;

(ii) there exists a director space $\bar{\Theta}$ of $\mathcal{D}_{L}$ such that

$$
\Gamma=\left\langle\bar{\Theta}, \bar{\Theta}^{\tau}, \ldots, \bar{\Theta}^{\tau^{t-3}}\right\rangle_{q^{t}},
$$

where $\tau=\Psi^{m}$ with $\operatorname{gcd}(m, t)=1$.

Proof Since each line $s$ of $\mathcal{P}$ has weight $t$ in $L$, it is clear that $\langle\Gamma, s\rangle_{q^{t}} \cap \Sigma$ is a $(t-1)$-dimensional subspace of $\Sigma$. Also, since the lines of $\mathcal{P}$ are pairwise disjoint and $|\mathcal{P}|=\frac{q^{n t}-1}{q^{t}-1}$, the set $\mathcal{D}_{L}$ in (i) is a $(t-1)$-spread of $\Sigma$.

Denote by $T_{1}$ and $T_{2}$ the transversal spaces of $\mathcal{P}$ and let $K_{1}$ be the $(n(t-1)-1)$ dimensional subspace of $\Sigma^{*}$ joining $\Gamma$ and $T_{1}$. Since $L$ is disjoint from $T_{1}$, we have that $K_{1} \cap \Sigma=\emptyset$, and hence $K_{1} \cap K_{1}^{\Psi} \cap \cdots \cap K_{1}^{\Psi^{t-1}}=\emptyset$. So $\Theta=K_{1} \cap K_{1}^{\Psi} \cap \cdots \cap$ $K_{1}^{\Psi^{t-2}}$ is an $(n-1)$-dimensional subspace of $\Sigma^{*}$ and $\Sigma^{*}=\left\langle\Theta, \Theta^{\Psi}, \ldots, \Theta^{\Psi^{t-1}}\right\rangle_{q^{t}}$.

Now, for each line $s$ of $\mathcal{P}$, let $X_{s}=\langle\Gamma, s\rangle_{q^{t}} \cap \Sigma$ be the corresponding element of the spread $\mathcal{D}_{L}$ and denote by $X_{s}^{*}$ the $(t-1)$-dimensional subspace of $\Sigma^{*}$ such that

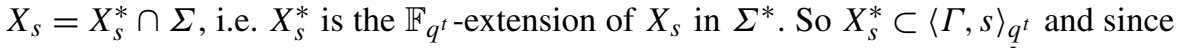
$X_{s}^{*}$ intersects $\Sigma$ in a subspace of the same dimension, we have that $\left(X_{s}^{*}\right)^{\Psi}=X_{s}^{*}$ (see [20, Lemma 1]). Also, let $P$ be the point $s \cap T_{1}$. Then $\langle\Gamma, P\rangle_{q^{t}}$ is a hyperplane of $\langle\Gamma, s\rangle_{q^{t}}$ and hence $H_{s}=\langle\Gamma, P\rangle_{q^{t}} \cap X_{s}^{*}$ is a $(t-2)$-dimensional subspace of $X_{s}^{*}$. Since $H_{s} \subseteq K_{1}$, we have that $H_{s}$ is disjoint from $\Sigma$ and hence $H_{s} \cap H_{s}^{\Psi} \cap \cdots \cap H_{s}^{\Psi^{t-1}}=\emptyset$. So $H_{s}, H_{s}^{\Psi}, \ldots, H_{s}^{\Psi^{t-1}}$ are $t$ independent hyperplanes of $X_{s}^{*}$. This implies that $H_{s} \cap$ $H_{s}^{\Psi} \cap \cdots \cap H_{s}^{\Psi^{t-2}}$ is a point, say $R_{S}$, of $X_{s}^{*}$. So

$$
R_{s} \in X_{s}^{*} \cap\left(H_{s} \cap H_{s}^{\Psi} \cap \cdots \cap H_{s}^{\Psi^{t-2}}\right) \subset X_{s}^{*} \cap\left(K_{1} \cap K_{1}^{\Psi} \cap \cdots \cap K_{1}^{\Psi^{t-2}}\right)=X_{s}^{*} \cap \Theta
$$

for each $s \in \mathcal{P}$. By Remark 2.1 we get that $\mathcal{D}_{L}$ is a Desarguesian spread of $\Sigma$ with $\Theta$ as a director space. Also, $\Theta^{\Psi^{i}} \subset K_{1}$ for each $i \neq 1$ and hence $K_{1}=\left\langle\Theta^{\Psi^{i}}: i \neq 1\right\rangle_{q^{t}}$ and $K_{1} \cap \Theta^{\Psi}=\emptyset$.

Similarly, if $K_{2}=\left\langle\Gamma, T_{2}\right\rangle_{q^{t}}$, we get that $K_{2} \cap K_{2}^{\Psi} \cap \cdots \cap K_{2}^{\Psi^{t-2}}$ is a director space of the Desarguesian spread $\mathcal{D}_{L}$ and hence there exists $m \in\{1,2, \ldots, t-1\}$ such that $K_{2} \cap K_{2}^{\Psi} \cap \cdots \cap K_{2}^{\Psi^{t-2}}=\Theta^{\Psi^{m}}$ (see [18, Theorem 3]). So $\Theta^{\Psi^{i}} \subset K_{2}$ for each $i \neq m+1$ and hence $K_{2}=\left\langle\Theta^{\Psi^{i}}: i \neq m+1\right\rangle_{q^{t}}$ and $K_{2} \cap \Theta^{\Psi^{m+1}}=\emptyset$. This means that

$$
\Gamma=K_{1} \cap K_{2}=\left\langle\Theta^{\Psi^{i}}: i \neq 1, m+1\right\rangle_{q^{t}} .
$$


So, if $\Psi^{m}=\tau$ and $\bar{\Theta}=\Theta^{\Psi^{2 m+1}}$, we get (8) of (ii). Finally, since $L$ is a scattered $\mathbb{F}_{q}$-linear set, by Theorem 3.9, Remarks 3.10 and 2.3, we have that $\operatorname{gcd}(t, m)=1$.

By Theorems 3.9 and 3.11 we have the following:

Theorem 3.12 Each $\mathbb{F}_{q}$-linear set of $P G\left(2 n-1, q^{t}\right)$ of pseudoregulus type is of the form $L_{\rho, f}$ described in Theorem 3.5.

Finally, by Theorem 3.12 and by Corollary 3.8 we can state the following classification result which generalizes [18, Theorem 4].

Theorem 3.13 In the projective space $\Lambda=P G\left(2 n-1, q^{t}\right)(n \geq 2, t \geq 3)$ there are $\varphi(t) / 2$ orbits of maximum scattered $\mathbb{F}_{q}$-linear sets of pseudoregulus type under the action of the collineation group of $\Lambda$.

\section{A class of maximum scattered $\mathbb{F}_{q}$-linear sets of $P G\left(1, q^{t}\right)$}

The arguments proving Theorem 3.5 can be exploited to construct a class of maximum scattered $\mathbb{F}_{q}$-linear sets of the projective line $\Lambda=P G\left(V, \mathbb{F}_{q^{t}}\right)=P G\left(1, q^{t}\right)$ with a structure resembling that of an $\mathbb{F}_{q}$-linear set of $P G\left(2 n-1, q^{t}\right)(n, t \geq 2)$ of pseudoregulus type. To this aim let $P_{1}=\langle\underline{w}\rangle_{q^{t}}$ and $P_{2}=\langle\underline{v}\rangle_{q^{t}}$ be two distinct points of $\Lambda$ and let $\tau$ be an $\mathbb{F}_{q}$-automorphism of $\mathbb{F}_{q^{t}}$ such that Fix $(\tau)=\mathbb{F}_{q}$; then for each $\rho \in \mathbb{F}_{q^{t}}^{*}$ the set

$$
W_{\rho, \tau}=\left\{\lambda \underline{w}+\rho \lambda^{\tau} \underline{v}: \lambda \in \mathbb{F}_{q^{t}}\right\},
$$

is an $\mathbb{F}_{q}$-vector subspace of $V$ of dimension $t$ and $L_{\rho, \tau}:=L_{W_{\rho, \tau}}$ is a scattered $\mathbb{F}_{q^{-}}$ linear set of $\Lambda$.

Definition 4.1 We call the linear sets $L_{\rho, \tau}$ of pseudoregulus type and we refer to the points $P_{1}$ and $P_{2}$ as transversal points of $L_{\rho, \tau}$.

If $L_{\rho, \tau} \cap L_{\rho^{\prime}, \tau} \neq \emptyset$, then $L_{\rho, \tau}=L_{\rho^{\prime}, \tau}$. Note that $L_{\rho, \tau}=L_{\rho^{\prime}, \tau}$ if and only if $N_{q^{t} / q}(\rho)=N_{q^{t} / q}\left(\rho^{\prime}\right)$; so $P_{1}, P_{2}$ and the automorphism $\tau$ define a set of $q-1$ mutually disjoint maximum scattered linear sets of pseudoregulus type admitting the same transversal points. Such maximal scattered linear sets, together with $P_{1}$ and $P_{2}$, cover the point set of the line $\Lambda=P G\left(1, q^{t}\right)$.

Remark 4.2 Since the group $P G L\left(2, q^{t}\right)$ acts 2-transitively on the points of $\Lambda$, we may suppose that all $\mathbb{F}_{q}$-linear sets of pseudoregulus type of $\Lambda$ have the same transversal points $P_{1}$ and $P_{2}$. This means that all such linear sets are only determined by $\rho$ and by the automorphism $\tau$. Moreover, it is easy to see that for each $\rho, \rho^{\prime} \in \mathbb{F}_{q^{t}}^{*}$ the linear sets $L_{\rho, \tau}$ and $L_{\rho^{\prime}, \tau}$ are equivalent. Indeed, it is sufficient to consider the collineation of $\Lambda=P G\left(V, \mathbb{F}_{q^{t}}\right)$ induced by the map $a \underline{w}+b \underline{v} \in V \mapsto$ $a \rho^{\tau^{-1}} \underline{w}+b \rho^{\prime} \underline{v} \in V$. It follows that, up to projectively equivalence, we may only consider $\mathbb{F}_{q}$-linear sets of type $L_{1, \sigma_{i}}$, where $\sigma_{i}: x \in \mathbb{F}_{q^{t}} \mapsto x^{q^{i}} \in \mathbb{F}_{q^{t}}$, with $i \in$ 
$\{1, \ldots, t-1\}$ and $\operatorname{gcd}(i, t)=1$. Now, by observing that, for each $i, j \in\{1, \ldots, t-1\}$ with $\operatorname{gcd}(i, t)=\operatorname{gcd}(j, t)=1$,

$$
\begin{aligned}
L_{1, \sigma_{i}} & =\left\{\left\langle\left(x, x^{q^{i}}\right)\right\rangle_{q^{t}}: x \in \mathbb{F}_{q^{t}}^{*}\right\}=\left\{\left\langle\left(1, x^{q^{i}-1}\right)\right\rangle_{q^{t}}: x \in \mathbb{F}_{q^{t}}^{*}\right\} \\
& =\left\{\langle(1, a)\rangle_{q^{t}}: a \in \mathbb{F}_{q^{t}}^{*}, N_{q^{t} / q}(a)=1\right\}=\left\{\left\langle\left(x, x^{q^{j}}\right)\right\rangle_{q^{t}}: x \in \mathbb{F}_{q^{t}}^{*}\right\}=L_{1, \sigma_{j}},
\end{aligned}
$$

we have that in $\Lambda=P G\left(1, q^{t}\right)(t \geq 2)$ all $\mathbb{F}_{q}$-linear sets of pseudoregulus type are equivalent to the linear set $L_{1, \sigma_{1}}$, under the action of the collineation group of $\Lambda$. This result has also been proven in [7, Remark 2.2].

Proposition 4.3 If $L$ is an $\mathbb{F}_{q}$-linear set of pseudoregulus type of $\Lambda=P G\left(1, q^{t}\right)$, $t \geq 3$, then its transversal points are uniquely determined.

Proof By Remark 4.2, we may consider the $\mathbb{F}_{q}$-linear set of pseudoregulus type

$$
L:=L_{1, \sigma_{1}}=\left\{\left\langle\left(\lambda, \lambda^{q}\right)\right\rangle_{q^{t}}: \lambda \in \mathbb{F}_{q^{t}}^{*}\right\},
$$

having $P_{1}=\langle(1,0)\rangle_{q^{t}}$ and $P_{2}=\langle(0,1)\rangle_{q^{t}}$ as transversal points.

Suppose that $L$ has another pair of transversal points $P_{1}^{\prime}=\langle\underline{w}\rangle_{q^{t}}$ and $P_{2}^{\prime}=\langle\underline{v}\rangle_{q^{t}}$, with $\underline{w}=\langle(a, b)\rangle_{q^{t}}$ and $\underline{v}=\langle(c, d)\rangle_{q^{t}}$, such that $a d \neq b c$. Then $L=\{\langle\eta \underline{w}+$ $\left.\left.\rho \eta^{\tau} \underline{v}\right\rangle_{q^{t}}: \eta \in \mathbb{F}_{q^{t}}^{*}\right\}$, with $\tau \in \operatorname{Aut}\left(\mathbb{F}_{q^{t}}\right)$. Moreover, arguing as in the previous remark, we have that

$$
\begin{aligned}
L & =\left\{\left\langle\underline{w}+\rho \eta^{\tau-1} \underline{v}\right\rangle_{q^{t}}: \eta \in \mathbb{F}_{q^{t}}^{*}\right\}=\left\{\left\langle\underline{w}+\rho \mu^{q-1} \underline{v}\right\rangle_{q^{t}}: \mu \in \mathbb{F}_{q^{t}}^{*}\right\} \\
& =\left\{\left\langle\mu \underline{w}+\rho \mu^{q} \underline{v}\right\rangle_{q^{t}}: \mu \in \mathbb{F}_{q^{t}}^{*} .\right.
\end{aligned}
$$

By (9) and (10), we have that for each $\lambda \in \mathbb{F}_{q^{t}}^{*}$, there exist $\alpha_{\lambda}, \mu \in \mathbb{F}_{q^{t}}^{*}$ such that

$$
\left(\lambda, \lambda^{q}\right)=\alpha_{\lambda}\left(\mu \underline{w}+\rho \mu^{q} \underline{v}\right)=\alpha_{\lambda}\left(\mu a+\rho \mu^{q} c, \mu b+\rho \mu^{q} d\right) .
$$

Then, the above equality implies that

$$
\alpha_{\lambda}^{q-1}=\frac{\mu b+\rho \mu^{q} d}{\left(\mu a+\rho \mu^{q} c\right)^{q}},
$$

which gives $N_{q^{t} / q}\left(\mu b+\rho \mu^{q} d\right)=N_{q^{t} / q}\left(\mu a+\rho \mu^{q} c\right)$ for each $\mu \in \mathbb{F}_{q^{t}}$, i.e.

$$
\prod_{i=0}^{t-1}\left(\mu^{q^{i}} b^{q^{i}}+\rho^{q^{i}} \mu^{q^{i+1}} d^{q^{i}}\right)=\prod_{i=0}^{t-1}\left(\mu^{q^{i}} a^{q^{i}}+\rho^{q^{i}} \mu^{q^{i+1}} c^{q^{i}}\right)
$$

for each $\mu \in \mathbb{F}_{q^{t}}$. From the last equality we get a polynomial identity in the variable $\mu$ of degree at most $2 q^{t-1}+q^{t-2}+\cdots+q^{3}+q^{2}+q$. If $q \geq 3$, then $2 q^{t-1}+q^{t-2}+$ $\cdots+q^{3}+q^{2}+q<q^{t}$, hence the polynomials in (11) are the same. So comparing the coefficients of the terms of maximum degree, we get

$$
d^{1+q+q^{2}+\cdots+q^{t-2}} b^{q^{t-1}}=c^{1+q+q^{2}+\cdots+q^{t-2}} a^{q^{t-1}} .
$$


Also, comparing the coefficients of the terms of degree $2 q^{t-1}+q^{t-2}+\cdots+q^{3}+$ $q^{2}+1$, for $t>2$, we have

$$
d^{q+q^{2}+\cdots+q^{t-2}} b^{q^{t-1}} b=c^{q+q^{2}+\cdots+q^{t-2}} a^{q^{t-1}} a .
$$

If $b d \neq 0$, then $a c \neq 0$ and dividing both sides of Eqs. (12) and (13), we get $\frac{d}{b}=\frac{c}{a}$, a contradiction since $P_{1}^{\prime} \neq P_{2}^{\prime}$. If $b=0$, from (12) we have $c=0$ and hence $P_{1}^{\prime}=P_{1}$ and $P_{2}^{\prime}=P_{2}$; if $d=0$, then also $a=0$ by (12) and hence $P_{1}^{\prime}=P_{2}$ and $P_{2}^{\prime}=P_{1}$.

If $q=2$, reducing (11) modulo $\mu^{q^{t}}-\mu$, we get that the two polynomials of (11) have degree at most $q^{t-1}+q^{t-2}+\cdots+q^{3}+q^{2}+q+1$. So, comparing the coefficients of the terms of degree $q^{t-1}+q^{t-2}+\cdots+q^{3}+q^{2}+2$, and of the terms of degree $q^{t-2}+q^{t-3}+\cdots+q^{3}+q^{2}+2$ (for $t>2$ ), and arguing as above we get the same result. This completes the proof.

Remark 4.4 Note that if $t=2$, then $L_{\rho, \tau}$ is a Baer subline of $\Lambda=P G\left(1, q^{2}\right)$ and $P_{1}$ and $P_{2}$ are conjugated with respect to the semilinear involution of $\Lambda$ fixing $L_{\rho, \tau}$ pointwise. Hence, in such a case, the transversal points are not uniquely determined.

Remark 4.5 Let $L_{\rho, f}$ be an $\mathbb{F}_{q}$-linear set of pseudoregulus type of $P G\left(2 n-1, q^{t}\right)$, $n>1$, and let $\mathcal{P}_{L_{\rho, f}}$ be the associated $\mathbb{F}_{q}$-pseudoregulus. By (3) and Definition 4.1, we observe that for each line $s \in \mathcal{P}_{\rho, f}$, the set $L_{\rho, f} \cap s$ is a linear set of pseudoregulus type whose transversal points are the intersections of $s$ with the transversal subspaces of $\mathcal{P}_{L_{\rho, f}}$.

We conclude this section by giving some examples of maximum scattered $\mathbb{F}_{q^{-}}$ linear sets which are not of pseudoregulus type.

\section{Example 4.6}

(i) Let

$$
L_{\rho}=\left\{\left\langle\left(x, \rho x^{q}+x^{q^{t-1}}\right)\right\rangle_{q^{t}}: x \in \mathbb{F}_{q^{t}}^{*}\right\},
$$

where $\rho \in \mathbb{F}_{q^{t}}$ such that $N_{q^{t} / q}(\rho) \neq 1$. By [22, Theorem 2] $L_{\rho}$ is a scattered $\mathbb{F}_{q^{-}}$ linear set of rank $t$. Moreover, if $q>3, \rho \neq 0$ and $t \geq 4$, by [22, Theorem 3], there is no collineation of $P G\left(1, q^{t}\right)$ mapping $L_{\rho}$ to $L_{1, \sigma_{1}}$. Hence, by Remark 4.2, $L_{\rho}$ is a maximum scattered $\mathbb{F}_{q}$-linear set which is not of pseudoregulus type when $q>3$.

(ii) Let

$$
L=\left\{\left\langle\left(x_{0}, x_{1}, \ldots, x_{n-1}, \rho x_{0}^{q}+x_{0}^{q^{t-1}}, x_{1}^{q}, \ldots, x_{n-1}^{q}\right)\right\rangle_{q^{t}}: x_{i} \in \mathbb{F}_{q^{t}}\right\},
$$

with $\rho \in \mathbb{F}_{q^{t}}^{*}$ and $N_{q^{t} / q}(\rho) \neq 1$. It is easy to see that $L$ is a scattered $\mathbb{F}_{q}$-linear set of rank $t n$. Also, the line $r$ with equations $x_{1}=x_{2}=\cdots=x_{n-1}=0$ is a line of weight $t$ in $L$ and, by the previous arguments $r \cap L$ is an $\mathbb{F}_{q}$-linear set which is not of pseudoregulus type for $q>3$. So by Remark 4.5 and by point (i), for each $q>3, t \geq 4$ and $n \geq 2, L$ is not of pseudoregulus type. 


\section{Linear sets and the variety $\Omega\left(\mathcal{S}_{n, n}\right)$}

Let $\mathbb{M}=\mathbb{M}(n, q)(n \geq 2)$ be the vector space of the matrices of order $n \times n$ with entries in $\mathbb{F}_{q}$ and let $P G\left(n^{2}-1, q\right)=P G\left(\mathbb{M}, \mathbb{F}_{q}\right)$. The Segre variety $\mathcal{S}_{n, n}=\mathcal{S}_{n, n}(q)$ of $P G\left(n^{2}-1, q\right)$ is the set of all points $\langle X\rangle_{q}$ of $P G\left(n^{2}-1, q\right)$ such that $X$ is a matrix of rank 1 . Here below we list some well known properties of such a variety, that can be found in [9, pp. 98-99], [10] and [11, Sect. 25.5]. Precisely,

- $\left|\mathcal{S}_{n, n}\right|=\left(\frac{q^{n}-1}{q-1}\right)^{2}$;

- maximal subspaces of $\mathcal{S}_{n, n}$ have dimension $n-1$;

- there are two families $\mathcal{R}_{1}$ and $\mathcal{R}_{2}$ of maximal subspaces of $\mathcal{S}_{n, n}$, which are the systems of $\mathcal{S}_{n, n}$. Spaces of the same system are pairwise skew and any two spaces of different systems meet in exactly one point. The elements of each system partition $\mathcal{S}_{n, n}$. Moreover, $\left|\mathcal{R}_{1}\right|=\left|\mathcal{R}_{2}\right|=\frac{q^{n}-1}{q-1}$

- the automorphism group $\operatorname{Aut}\left(\mathcal{S}_{n, n}\right)$ of $\mathcal{S}_{n, n}$ is isomorphic to $(P \Gamma L(n, q) \times$ $P \Gamma L(n, q)) \rtimes C_{2}$, and it is the group of all collineations of $P G\left(n^{2}-1, q\right)$ fixing or interchanging the two systems of $\mathcal{S}_{n, n}$.

A $k$-dimensional subspace $S$ of $P G\left(n^{2}-1, q\right)$ is a $k$ th secant subspace to $\mathcal{S}_{n, n}$ when $S=\left\langle P_{1}, P_{2}, \ldots, P_{k+1}\right\rangle_{q}$ and $\left\{P_{1}, P_{2}, \ldots, P_{k+1}\right\} \subset S \cap \mathcal{S}_{n, n}$. The $(n-2)$ th secant variety $\Omega\left(\mathcal{S}_{n, n}\right)$ of $\mathcal{S}_{n, n}$ is the set of all points of $P G\left(n^{2}-1, q\right)$ belonging to an $(n-2)$ th secant subspace to $\mathcal{S}_{n, n}$. Note that

$$
\Omega\left(\mathcal{S}_{n, n}\right)=\left\{\langle X\rangle_{q} \mid X \in \mathbb{M}(n, q) \backslash\{\mathbf{0}\}, \operatorname{det} X=0\right\},
$$

i.e. $\Omega\left(\mathcal{S}_{n, n}\right)$ is the algebraic variety, also called determinantal hypersurface, defined by the non-invertible matrices of $\mathbb{M}(n, q)$.

Regarding $\mathbb{F}_{q^{n}}$ as an $n$-dimensional vector space over $\mathbb{F}_{q}$ and fixing an $\mathbb{F}_{q}$-basis $\mathcal{B}$ of $\mathbb{F}_{q^{n}}$, each matrix $M$ of $\mathbb{M}=\mathbb{M}(n, q)$ defines an $\mathbb{F}_{q}$-endomorphism $\varphi_{M}$ of $\mathbb{F}_{q^{n}}$, and conversely. The map $\phi_{M}: M \in \mathbb{M} \mapsto \varphi_{M} \in \mathbb{E}$, where $\mathbb{E}=\operatorname{End}\left(\mathbb{F}_{q^{n}}, \mathbb{F}_{q}\right)$ is the $n^{2}$-dimensional vector space of all the $\mathbb{F}_{q}$-endomorphisms of $\mathbb{F}_{q^{n}}$, is an isomorphism between the vector spaces $\mathbb{M}$ and $\mathbb{E}$. By using such an isomorphism, we have that the elements of $\mathbb{E}$ with rank 1 define in $P G\left(\mathbb{E}, \mathbb{F}_{q}\right)=P G\left(n^{2}-1, q\right)$ the Segre variety $\mathcal{S}_{n, n}$. Recalling that each element $\varphi \in \mathbb{E}$ can be written as $\varphi(x)=\sum_{i=0}^{n-1} \beta_{i} x^{q^{i}}$, with $\beta_{i} \in \mathbb{F}_{q^{n}}$, we get the following result.

Proposition 5.1 Let $\mathbb{P}=P G\left(\mathbb{E}, \mathbb{F}_{q}\right)=P G\left(n^{2}-1, q\right)$ and let $\mathcal{S}_{n, n}$ be the Segre variety of $\mathbb{P}$ defined by the elements of $\mathbb{E}$ with rank 1 . Then 4

$$
\mathcal{S}_{n, n}=\left\{\left\langle t_{\lambda} \circ \operatorname{Tr} \circ t_{\mu}\right\rangle_{q}: \lambda, \mu \in \mathbb{F}_{q^{n}}^{*}\right\},
$$

where $t_{\alpha}: x \in \mathbb{F}_{q^{n}} \mapsto \alpha x \in \mathbb{F}_{q^{n}}$, with $\alpha \in \mathbb{F}_{q^{n}}$ and $\operatorname{Tr}: x \in \mathbb{F}_{q^{n}} \mapsto x+x^{q}+\cdots+$ $x^{q^{n-1}} \in \mathbb{F}_{q}$. Moreover, $\mathcal{R}_{1}=\left\{X(\lambda): \lambda \in \mathbb{F}_{q^{n}}^{*}\right\}$ and $\mathcal{R}_{2}=\left\{X^{\prime}(\lambda): \lambda \in \mathbb{F}_{q^{n}}^{*}\right\}$, where

$$
\begin{aligned}
& X(\lambda)=\left\{\left\langle t_{\alpha} \circ \operatorname{Tr} \circ t_{\lambda}\right\rangle_{q}: \alpha \in \mathbb{F}_{q^{n}}^{*}\right\} \text { and } \\
& X^{\prime}(\lambda)=\left\{\left\langle t_{\lambda} \circ \operatorname{Tr} \circ t_{\alpha}\right\rangle_{q}: \alpha \in \mathbb{F}_{q^{n}}^{*},\right.
\end{aligned}
$$

4 o stands for composition of maps. 
are the two systems of $\mathcal{S}_{n, n}$. Finally, $\Omega\left(\mathcal{S}_{n, n}\right)$ is defined by the non-invertible elements of $\mathbb{E}$.

Proof Note that, for each $\lambda, \mu \in \mathbb{F}_{q^{n}}^{*}$, we have

$$
\operatorname{ker}\left(t_{\lambda} \circ \operatorname{Tr} \circ t_{\mu}\right)=\frac{1}{\mu} \operatorname{ker} \operatorname{Tr},
$$

so $\operatorname{dim}\left(\operatorname{ker}\left(t_{\lambda} \circ \operatorname{Tr} \circ t_{\mu}\right)\right)=n-1$ (i.e., $t_{\lambda} \circ \operatorname{Tr} \circ t_{\mu}$ is an element of $\mathbb{E}$ of rank 1$)$ and hence $\left\langle t_{\lambda} \circ \operatorname{Tr} \circ t_{\mu}\right\rangle_{q} \in \mathcal{S}_{n, n}$. Also, for each $\lambda^{\prime}, \mu^{\prime} \in \mathbb{F}_{q^{n}}^{*}, t_{\lambda} \circ \operatorname{Tr} \circ t_{\mu}=t_{\lambda^{\prime}} \circ \operatorname{Tr} \circ t_{\mu^{\prime}}$ if and only if $\frac{\lambda}{\lambda^{\prime}}=\frac{\mu}{\mu^{\prime}} \in \mathbb{F}_{q}^{*}$. Then direct computations show that $\mid\left\{\left\langle t_{\lambda} \circ \operatorname{Tr} \circ t_{\mu}\right\rangle_{q}\right.$ : $\left.\lambda, \mu \in \mathbb{F}_{q^{n}}^{*}\right\} \mid=\left(\frac{q^{n}-1}{q-1}\right)^{2}$, and hence $\mathcal{S}_{n, n}=\left\{\left\langle t_{\lambda} \circ \operatorname{Tr} \circ t_{\mu}\right\rangle_{q}: \lambda, \mu \in \mathbb{F}_{q^{n}}^{*}\right\}^{5}$

Also, it is easy to prove that for each $\lambda \in \mathbb{F}_{q^{n}}^{*}$, the sets $X(\lambda)$ and $X^{\prime}(\lambda)$ are $(n-1)$ dimensional subspaces of $\mathbb{P}$ contained in $\mathcal{S}_{n, n}$. Moreover, for each $\lambda, \mu \in \mathbb{F}_{q^{n}}^{*}$, two subspaces $X(\lambda)$ and $X(\mu)$ are either disjoint or equal, and this latter case holds true if and only if $\frac{\lambda}{\mu} \in \mathbb{F}_{q}^{*}$. The same happens for $X^{\prime}(\lambda)$ and $X^{\prime}(\mu)$. This implies that $\mid\{X(\lambda)$ : $\left.\lambda \in \mathbb{F}_{q^{n}}^{*}\right\}|=|\left\{X^{\prime}(\lambda): \lambda \in \mathbb{F}_{q^{n}}^{*}\right\} \mid=\frac{q^{n}-1}{q-1}$. Also, $X(\lambda) \cap X^{\prime}(\mu)=\left\{\left\langle t_{\mu} \circ \operatorname{Tr} \circ t_{\lambda}\right\rangle_{q}\right\}$ is a point. Then $\mathcal{R}_{1}=\left\{X(\lambda): \lambda \in \mathbb{F}_{q^{n}}^{*}\right\}$ and $\mathcal{R}_{2}=\left\{X^{\prime}(\lambda): \lambda \in \mathbb{F}_{q^{n}}^{*}\right\}$ are the systems of $\mathcal{S}_{n, n}$. Finally, by (14) the last part of the assertion follows.

For each $\varphi \in \mathbb{E}$, where $\varphi(x)=\sum_{i=0}^{n-1} \beta_{i} x^{q^{i}}$, the conjugate $\bar{\varphi}$ of $\varphi$ is defined by $\bar{\varphi}(x)=\sum_{i=0}^{n-1} \beta_{i}^{q^{n-i}} x^{q^{n-i}}$. Precisely, $\bar{\varphi}$ is the adjoint map of $\varphi$ with respect to the non-degenerate bilinear form of $\mathbb{F}_{q^{n}}$

$$
\beta(x, y)=\operatorname{Tr}_{q^{n} / q}(x y) .
$$

The map

$$
T: \varphi \in \mathbb{E} \mapsto \bar{\varphi} \in \mathbb{E},
$$

is an involutory $\mathbb{F}_{q}$-linear permutation of $\mathbb{E}$ and straightforward computations show that

$$
\begin{aligned}
& \overline{\varphi \circ \psi}=\bar{\psi} \circ \bar{\varphi}, \quad \overline{\varphi^{-1}}=(\bar{\varphi})^{-1} \quad \text { for each } \varphi, \psi \in \mathbb{E} ; \\
& \overline{t_{\lambda}}=t_{\lambda} \quad \text { for each } \lambda \in \mathbb{F}_{q^{n}} .
\end{aligned}
$$

Moreover, it can be easily checked that $\operatorname{ker} \varphi=(\operatorname{Im} \bar{\varphi})^{\perp}$, where $\perp$ is the polarity defined by $(15)$, and hence $\operatorname{dim}(\operatorname{ker} \varphi)=\operatorname{dim}(\operatorname{ker} \bar{\varphi})$. Then $T$ induces in $\mathbb{P}$ a linear involutory collineation $\Phi_{T}$ preserving the varieties $\mathcal{S}_{n, n}$ and $\Omega\left(\mathcal{S}_{n, n}\right)$ and interchanging the systems $\mathcal{R}_{1}$ and $\mathcal{R}_{2}$ of $\mathcal{S}_{n, n}$. Indeed, we have

$$
X(\mu)^{\Phi_{T}}=X^{\prime}(\mu) \text { for each } \mu \in \mathbb{F}_{q^{n}}^{*} .
$$

\footnotetext{
${ }^{5}$ Alternatively, by [19, Theorem 2.24] it can be easily seen that the maps $t_{\lambda} \circ \operatorname{Tr} \circ t_{\mu}$ are all the $\mathbb{F}_{q^{-}}$ endomorphisms of $\mathbb{F}_{q^{n}}$ with rank 1 .
} 
The subgroup $H\left(\mathcal{S}_{n, n}\right)$ of $P \Gamma L\left(n^{2}, q\right)$ fixing the systems $\mathcal{R}_{1}$ and $\mathcal{R}_{2}$ of $\mathcal{S}_{n, n}$ is isomorphic to $P \Gamma L(n, q) \times P \Gamma L(n, q)$, and such a group has index 2 in the group $\operatorname{Aut}\left(\mathcal{S}_{n, n}\right)=\operatorname{Aut}\left(\Omega\left(\mathcal{S}_{n, n}\right)\right) \simeq(P \Gamma L(n, q) \times P \Gamma L(n, q)) \rtimes C_{2}$ (see [13, Theorem 3] and [11]). Hence $\operatorname{Aut}\left(\mathcal{S}_{n, n}\right)=\left\langle H\left(\mathcal{S}_{n, n}\right), \Phi_{T}\right\rangle$.

Let $\mathcal{I}:=\left\{\left\langle t_{\lambda}\right\rangle_{q}: \lambda \in \mathbb{F}_{q^{n}}^{*}\right\}$. Then $\mathcal{I}$ is an $(n-1)$-dimensional subspace of $\mathbb{P}$ disjoint from the variety $\Omega\left(\mathcal{S}_{n, n}\right)$ and

$$
\mathcal{D}_{1}(\mathcal{I})=\left\{\left\{\left\langle t_{\alpha} \circ \varphi\right\rangle_{q}: \alpha \in \mathbb{F}_{q^{n}}^{*}\right\}: \varphi \in \mathbb{E} \backslash\{\mathbf{0}\}\right\}
$$

and

$$
\mathcal{D}_{2}(\mathcal{I})=\left\{\left\{\left\langle\varphi \circ t_{\alpha}\right\rangle_{q}: \alpha \in \mathbb{F}_{q^{n}}^{*}\right\}: \varphi \in \mathbb{E} \backslash\{\mathbf{0}\}\right\}
$$

are two Desarguesian spreads of $\mathbb{P}$ (see, e.g., [3, Exercise 3 and Theorem 14]) such that

$\left(I_{1}\right) \mathcal{I} \in \mathcal{D}_{i}(\mathcal{I})$ and $\mathcal{R}_{i} \subset \mathcal{D}_{i}(\mathcal{I})$, for each $i \in\{1,2\}$.

Also, we explicitly note that

(I $I_{2} \Phi_{T}$ fixes $\mathcal{I}$ pointwise and, by $(18), \mathcal{D}_{1}(\mathcal{I})^{\Phi_{T}}=\mathcal{D}_{2}(\mathcal{I})$.

Let $\Pi_{n-1}\left(\mathcal{D}_{1}(\mathcal{I})\right)$ be the $\mathbb{F}_{q}$-linear representation of the projective space $\operatorname{PG}(n-$ $1, q^{n}$ ) defined by the Desarguesian spread $\mathcal{D}_{1}(\mathcal{I})$ of $\mathbb{P}$. Let $\Upsilon_{1}$ be the linear collineation of $\mathbb{P}$ defined as

$$
\Upsilon_{1}:\langle\varphi\rangle_{q} \in \mathbb{P} \mapsto\left\langle\varphi^{\prime}\right\rangle_{q} \in \mathbb{P}
$$

where $\varphi^{\prime}(x)=\sum_{i=0}^{n-1} a_{i-1}^{q} x^{q^{i}}$ if $\varphi(x)=\sum_{i=0}^{n-1} a_{i} x^{q^{i}}$, taking the indices $i$ modulo $n$.

( $\left.I_{3}\right)$ The collineation $\Upsilon_{1}$ fixes the Desarguesian spread $\mathcal{D}_{1}(\mathcal{I})$ and induces a collineation $\bar{\Upsilon}_{1}$ in $\Pi_{n-1}\left(\mathcal{D}_{1}(\mathcal{I})\right)$ of order $n$ whose fixed point set consists of the elements of $\mathcal{R}_{1}$. Hence, $\mathcal{R}_{1}$ turns out to be a subgeometry of $\Pi_{n-1}\left(\mathcal{D}_{1}(\mathcal{I})\right)$ isomorphic to $P G(n-1, q)$.

We explicitly note that

$$
\mathcal{I}^{\Upsilon_{1}^{j}}=\left\{\left\langle x \mapsto \lambda x^{q^{j}}\right\rangle_{q}: \lambda \in \mathbb{F}_{q^{n}}^{*}\right\} \quad \text { and } \quad\left\langle\mathcal{I}, \mathcal{I}^{\Upsilon_{1}}, \ldots, \mathcal{I}^{\Upsilon_{1}^{n-1}}\right\rangle_{q}=\mathbb{P}
$$

So $\mathcal{I}$, in $\Pi_{n-1}\left(\mathcal{D}_{1}(\mathcal{I})\right)$, is a point whose orbit under the action of the cyclic group $\left\langle\bar{\Upsilon}_{1}\right\rangle$ has maximum size $n$.

In the same way,

( $\left.I_{4}\right)$ the collineation $\Upsilon_{2}=\Phi_{T}^{-1} \circ \Upsilon_{1} \circ \Phi_{T}$ fixes the Desarguesian spread $\mathcal{D}_{2}(\mathcal{I})$ and induces a collineation $\bar{\Upsilon}_{2}$ in $\Pi_{n-1}\left(\mathcal{D}_{2}(\mathcal{I})\right)$ of order $n$ whose set of fixed points consists of the elements of $\mathcal{R}_{2}$.

Also,

(I5) $\mathcal{I}^{\Upsilon_{2}^{i}}=\mathcal{I}^{\Upsilon_{1}^{n-i}}$. 
Let $\mathcal{O}_{\mathcal{I}}$ be the orbit, under the action of the group $H\left(\mathcal{S}_{n, n}\right)$, of the $(n-1)$-dimensional subspace $\mathcal{I}$ of $\mathbb{P}$. A subspace belonging to this orbit will be called a $\mathcal{D}$-subspace of $\mathbb{P}$. In the following we will study the geometric properties of the $\mathcal{D}$-subspaces of $\mathbb{P}$ under the action of $H\left(\mathcal{S}_{n, n}\right)$.

Theorem 5.2 Let $X$ be a $\mathcal{D}$-subspace of $\mathbb{P}=P G\left(\mathbb{E}, \mathbb{F}_{q}\right)=P G\left(n^{2}-1, q\right)$, then there exist two Desarguesian spreads $\mathcal{D}_{1}(X)$ and $\mathcal{D}_{2}(X)$ of $\mathbb{P}$ such that:

$\left(D_{1}\right) \quad X \in \mathcal{D}_{i}(X)$ and $\mathcal{R}_{i} \subset \mathcal{D}_{i}(X)$ for each $i=1,2$,

$\left(D_{2}\right)$ there is a semilinear collineation $\bar{\Xi}_{i}$ of $\Pi_{n-1}\left(\mathcal{D}_{i}(X)\right)$ of order $n$ induced by a linear collineation $\Xi_{i}$ of $\mathbb{P}$ fixing the Desarguesian spread $\mathcal{D}_{i}(X)$. Moreover, $\mathcal{R}_{i}=$ Fix $\bar{\Xi}_{i}$ is a subgeometry of $\Pi_{n-1}\left(\mathcal{D}_{i}(X)\right)$ isomorphic to a $\operatorname{PG}(n-1, q)$.

Also, there exists an involutory collineation $\Phi$ of $\mathbb{P}$ such that

$\left(D_{3}\right) \Phi$ fixes $X$ pointwise,

$\left(D_{4}\right) \mathcal{D}_{1}(X)^{\Phi}=\mathcal{D}_{2}(X)$.

Proof Let $g$ be an element of $H\left(\mathcal{S}_{n, n}\right)$ such that $\mathcal{I}^{g}=X$. By $\left(I_{1}\right), \mathcal{D}_{i}(X):=\mathcal{D}_{i}(\mathcal{I})^{g}$, for each $i \in\{1,2\}$, is a Desarguesian spread of $\mathbb{P}$ containing $X$ and the system $\mathcal{R}_{i}$, i.e. $\left(D_{1}\right)$ is satisfied. Putting $\Xi_{i}:=g \circ \Upsilon_{i} \circ g^{-1}$ and $\Phi:=g \circ \Phi_{T} \circ g^{-1}$ and taking $\left(I_{2}\right),\left(I_{3}\right)$ and $\left(I_{4}\right)$ into account, $\left(D_{2}\right),\left(D_{3}\right)$ and $\left(D_{4}\right)$ follow.

This allows us to give the following

Definition 5.3 Let $X$ be a $\mathcal{D}$-subspace of $\mathbb{P}$ and let $\Xi_{i}(i \in\{1,2\})$ be one of the two collineations of $\mathbb{P}$ described in $\left(D_{2}\right)$. Each of the $\mathcal{D}$-subspaces $X^{\Xi_{i}^{j}}$, with $j \in$ $\{0,1, \ldots, n-1\}$, is said to be a conjugate of $X$. Note that, by $\left(I_{5}\right), X^{\Xi_{2}^{j}}=X^{\Xi_{1}^{n-j}}$.

Remark 5.4 If $n=2$, then $\mathbb{E}=\operatorname{End}\left(\mathbb{F}_{q^{2}}, \mathbb{F}_{q}\right)$ and $\mathcal{S}_{2,2}$ is the hyperbolic quadric $Q^{+}(3, q)$ of $\mathbb{P}=P G\left(\mathbb{E}, \mathbb{F}_{q}\right)=P G(3, q)$ defined by the quadratic form

$$
\varphi \in \mathbb{E} \mapsto a^{q+1}-b^{q+1} \in \mathbb{F}_{q},
$$

where $\varphi(x)=a x+b x^{q}$. Hence, the group $H\left(\mathcal{S}_{2,2}\right)$ is the subgroup of the orthogonal group $P \Gamma O^{+}(4, q)$ fixing the reguli of $Q^{+}(3, q)$. Also, the $H\left(\mathcal{S}_{2,2}\right)$-orbit of the line $\mathcal{I}$, is the set of all external lines to the quadric. Moreover, the involutory linear collineation $\Upsilon_{1}$ of $\mathbb{P}$ described above is

$$
\left\langle x \mapsto a x+b x^{q}\right\rangle_{q} \longmapsto\left\langle x \mapsto b^{q} x+a^{q} x^{q}\right\rangle_{q}
$$

This means that the conjugate of $\mathcal{I}$ is the line $\mathcal{I}^{\Upsilon_{1}}=\left\{\left\langle x \mapsto \mu x^{q}\right\rangle_{q}: \mu \in \mathbb{F}_{q^{2}}^{*}\right\}$, which is the polar line of $\mathcal{I}$ with respect to the quadric $Q^{+}(3, q)$.

\subsection{Linear sets and presemifields}

A finite semifield is a finite division algebra which is not necessarily associative and throughout this paper the term semifield will always be used to denote a finite semifield (see, e.g., [16, Chap. 6] for definitions and notations on finite semifields). Every 
field is a semifield and the term proper semifield means a semifield which is not a field. The left nucleus $\mathbb{N}_{l}$ and the center $\mathbb{K}$ of a semifield $\mathbb{S}$ are fields contained in $\mathbb{S}$ as substructures ( $\mathbb{K}$ is a subfield of $\mathbb{N}_{l}$ ) and $\mathbb{S}$ is a vector space over $\mathbb{N}_{l}$ and over $\mathbb{K}$. Semifields are studied up to an equivalence relation called isotopy and the dimensions of a semifield over its left nucleus and over its center are invariant up to isotopy.

Let $\mathbb{S}$ be a semifield with center $\mathbb{K}$ and left nucleus $\mathbb{N}_{l}$ and let $\left(\mathbb{F}_{q}, \mathbb{F}_{q^{t}}\right)$ be a pair of fields such that $\mathbb{F}_{q} \leq \mathbb{K}$ and $\mathbb{F}_{q^{t}} \leq \mathbb{N}_{l}$; then $\mathbb{S}$ is a finite extension of $\mathbb{F}_{q^{t}}$ and hence it has size $q^{n t}$ for some integer $n \geq 1$. If $\mathbb{S}$ is a proper semifield, then $n \geq 2$. Also, up to isotopy, we may assume that $\mathbb{S}=\left(\mathbb{F}_{q^{n t}},+, \star\right)$, where

$$
x \star y=\varphi_{y}(x)
$$

with $\varphi_{y} \in \mathbb{E}=\operatorname{End}\left(\mathbb{F}_{q^{n t}}, \mathbb{F}_{q^{t}}\right)$. The set

$$
\mathcal{C}_{\mathbb{S}}=\left\{\varphi_{y}: x \in \mathbb{F}_{q^{n t}} \mapsto x \star y \in \mathbb{F}_{q^{n t}} \mid y \in \mathbb{F}_{q^{n t}}\right\} \subset \mathbb{E}
$$

is the semifield spread set associated with $\mathbb{S}$ (spread set for short): $\mathcal{C}_{\mathbb{S}}$ is an $\mathbb{F}_{q^{-}}$ subspace of $\mathbb{E}$ of rank $n t$ and each non-zero element of $\mathcal{C}_{\mathbb{S}}$ is invertible. Hence, for each pair $\left(\mathbb{F}_{q}, \mathbb{F}_{q^{t}}\right) \subseteq\left(\mathbb{K}_{,} \mathbb{N}_{l}\right)$, we can associate with $\mathbb{S}$ the $\mathbb{F}_{q}$-linear set of rank $n t$ of the projective space $\mathbb{P}=P G\left(\mathbb{E}, \mathbb{F}_{q^{t}}\right)=P G\left(n^{2}-1, q^{t}\right)$ defined by the non-zero elements of $\mathcal{C}_{\mathbb{S}}$. Such a linear set turns out to be disjoint from the variety $\Omega\left(\mathcal{S}_{n, n}\left(q^{t}\right)\right)$ of $\mathbb{P}$ defined by the non-invertible elements of $\mathbb{E}$. Isotopic semifields produce in $\mathbb{P}=P G\left(n^{2}-1, q^{t}\right)$ linear sets which are equivalent with respect to the action of the group $H\left(\mathcal{S}_{n, n}\left(q^{t}\right)\right)$, and conversely (see [21] for $n=2$ and [14] for $n \geq 2$ ). Among all the pairs $\left(\mathbb{F}_{q}, \mathbb{F}_{q^{t}}\right)$ such that $\mathbb{F}_{q} \subseteq \mathbb{K}_{\text {and }} \mathbb{F}_{q^{t}} \subseteq \mathbb{N}_{l}$, the pair $\left(\mathbb{K}, \mathbb{N}_{l}\right)$ has the following properties: (i) maximizes the field of linearity of the linear set associated with $\mathbb{S}$, (ii) minimizes the dimension of the projective space $\mathbb{P}$ in which the linear set is embedded, and (iii) minimizes the group $H\left(\mathcal{S}_{n, n}\right)$. For instance, if $\mathbb{S}=\mathbb{F}_{q}$, then $\mathbb{N}_{l}=\mathbb{K}=\mathbb{F}_{q}$ and hence the linear set associated with the field $\mathbb{F}_{q}$, with respect to the pair $\left(\mathbb{F}_{q}, \mathbb{F}_{q}\right)$, is the point $P G\left(\mathbb{F}_{q}, \mathbb{F}_{q}\right)$; whereas, if $\mathbb{F}_{q^{\prime}}$ is a subfield of $\mathbb{F}_{q}, q=q^{\prime n}$, then the linear set associated with $\mathbb{F}_{q}$, with respect to the pair $\left(\mathbb{F}_{q^{\prime}}, \mathbb{F}_{q^{\prime}}\right)$, is an $(n-1)$ dimensional subspace of $\mathbb{P}=P G\left(n^{2}-1, q^{\prime}\right)$ disjoint from the variety $\Omega\left(\mathcal{S}_{n, n}\left(q^{\prime}\right)\right)$ of $\mathbb{P}$, which is a $\mathcal{D}$-subspace of $\mathbb{P}$, and conversely (see [13, Theorem 20]). In what follows, we will call the linear set associated with $\mathbb{S}$ with respect to the maximum pair $\left(\mathbb{K}, \mathbb{N}_{l}\right)$, the relevant linear set associated with $\mathbb{S}$.

In the next sections we will characterize, up to the action of the group $H\left(\mathcal{S}_{n, n}\right)$, the relevant linear sets associated with some classical semifields: the Generalized Twisted Fields and the Knuth semifields 2-dimensional over their left nucleus.

\subsection{Generalized Twisted Fields}

If $\mathbb{S}$ satisfies all the axioms for a semifield except, possibly, the existence of the identity element for the multiplication, then it is a presemifield. In such a case the nuclei and the center of $\mathbb{S}$ are defined as fields of linear maps contained in $\operatorname{End}\left(\mathbb{S}, \mathbb{F}_{p}\right.$ ) (where $p$ is the characteristic of $\mathbb{S}$ ) (see, e.g., [24, Theorem 2.2]) and all that we stated and defined above for semifields can be applied to presemifields. 
The Generalized Twisted Fields are presemifields constructed by A.A. Albert in [2]. By [1, Lemma 1] a Generalized Twisted Field $\mathbb{G}$ with center of order $q$, $n$ dimensional over its left nucleus $(n \geq 2)$ and $t n$-dimensional over its center is of type $\mathbb{G}=\left(\mathbb{F}_{q^{n t}},+, \star\right)\left(q=p^{e}, p\right.$ prime $)$ with

$$
x \star y=y x-c y^{q^{m}} x^{q^{t l}},
$$

where $c \in \mathbb{F}_{q^{n t}}^{*}, c \neq x^{q^{t l}-1} y^{q^{m}-1}$ for every $x, y \in \mathbb{F}_{q^{n t}}$, and $1 \leq l \leq n-1,1 \leq m \leq$ $n t-1, m \neq t l$. Since we required $\operatorname{dim}_{\mathbb{K}} \mathbb{G}=n t$ and $\operatorname{dim}_{\mathbb{N}_{l}} \mathbb{G}=n$, we also have $\operatorname{gcd}(l, n)=\operatorname{gcd}(t, m)=1$. From the previous conditions we get $q>2$ and, if $t=1$, then $n \geq 3$. In terms of linear maps, by [24, Theorem 2.2] and by [1, Lemma 1] we can describe the left nucleus and the center of $\mathbb{G}$ as follows:

$$
\begin{aligned}
\mathbb{N}_{l} & =\left\{t_{\lambda}: x \in \mathbb{F}_{q^{n t}} \mapsto \lambda x \in \mathbb{F}_{q^{n t}} \mid \lambda \in \mathbb{F}_{q^{t}}\right\} \subset \mathbb{E}, \\
\mathbb{K} & =\left\{t_{\lambda}: x \in \mathbb{F}_{q^{n t}} \mapsto \lambda x \in \mathbb{F}_{q^{n t}} \mid \lambda \in \mathbb{F}_{q}\right\} \subset \mathbb{E},
\end{aligned}
$$

where $\mathbb{E}=\operatorname{End}\left(\mathbb{F}_{q^{n t}}, \mathbb{F}_{q^{t}}\right)=V\left(n^{2}, \mathbb{F}_{q^{t}}\right)$. The spread set associated with $\mathbb{G}$ is

$$
\mathbb{C}=\left\{\varphi_{y}: x \in \mathbb{F}_{q^{n t}} \mapsto x \star y \in \mathbb{F}_{q^{n t}} \mid y \in \mathbb{F}_{q^{n t}}\right\} \subset \mathbb{E}
$$

and it is an $\mathbb{F}_{q}$-subspace of $\mathbb{E}$ of dimension $n t$. Hence $\mathbb{C}$ defines an $\mathbb{F}_{q}$-linear set of rank $n t$ in the projective space $\mathbb{P}=P G\left(\mathbb{E}, \mathbb{F}_{q^{t}}\right)=P G\left(n^{2}-1, q^{t}\right)$; precisely

$$
L(\mathbb{G})=L_{\mathbb{C}}=\left\{\left\langle\varphi_{y}\right\rangle_{q^{t}}: y \in \mathbb{F}_{q^{n t}}^{*}\right\},
$$

which is the relevant linear set associated with $\mathbb{G}$. Since the non-zero elements of $\mathbb{C}$ are invertible, $L(\mathbb{G})$ is disjoint from the variety $\Omega\left(\mathcal{S}_{n, n}\left(q^{t}\right)\right)$ of $\mathbb{P}$ defined by the noninvertible elements of $\mathbb{E}$. By $(20)$ it is clear that $L(\mathbb{G})$ is contained in the subspace $\Lambda=P G\left(2 n-1, q^{t}\right)$ of $\mathbb{P}$ joining the $\mathcal{D}$-space $\mathcal{I}=\left\{\left\langle t_{\lambda}\right\rangle_{q^{t}}: \lambda \in \mathbb{F}_{q^{n t}}^{*}\right\}$ and its conjugate $\mathcal{I}^{\Upsilon_{1}^{l}}=\left\{\left\langle x \mapsto \lambda x^{q^{t l}}\right\rangle_{q^{t}}: \lambda \in \mathbb{F}_{q^{n t}}^{*}\right\}$, precisely

$$
\Lambda=\left\{\left\langle x \mapsto A x+B x^{q^{l t}}\right\rangle_{q^{t}}: A, B \in \mathbb{F}_{q^{n t}}\right\} .
$$

Note that $\Lambda$ defines a line $P G\left(1, q^{n t}\right)$ in the $\mathbb{F}_{q^{t}}$-linear representation $\Pi_{n-1}\left(\mathcal{D}_{1}(\mathcal{I})\right)$. Also, since $\operatorname{gcd}(t, m)=1$, it is easy to verify that, if $t \geq 2$, then $L(\mathbb{G})$ is a maximum scattered $\mathbb{F}_{q}$-linear set of $\Lambda$ and, hence, $\Lambda=\langle L(\mathbb{G})\rangle_{q^{t}}$.

Proposition 5.5 Let $\mathbb{G}=\left(\mathbb{F}_{q^{n t}},+, \star\right)$ be a Generalized Twisted Field $n$-dimensional over its left nucleus and tn-dimensional over its center. Let $\mathbb{P}=P G\left(\mathbb{E}, \mathbb{F}_{q^{t}}\right)=$ $P G\left(n^{2}-1, q^{t}\right)\left(\right.$ where $\left.\mathbb{E}=\operatorname{End}\left(\mathbb{F}_{q^{n t}}, \mathbb{F}_{q^{t}}\right)\right), \Lambda=\left\{\left\langle x \mapsto A x+B x^{q^{l t}}\right\rangle_{q^{t}}: A, B \in \mathbb{F}_{q^{n t}}\right\}$ and $\Pi_{n-1}\left(\mathcal{D}_{1}(\mathcal{I})\right)$ be the $\mathbb{F}_{q}$-linear representation of $P G\left(n-1, q^{n}\right)$.

(a) If $t=1$, then $(a . i) L(\mathbb{G})$ is an $(n-1)$-dimensional subspace of $\mathbb{P}=P G\left(n^{2}-\right.$ $1, q)$ contained in $\Lambda$ and in the linear representation $\Pi_{n-1}\left(\mathcal{D}_{1}(\mathcal{I})\right) \simeq P G(n-$

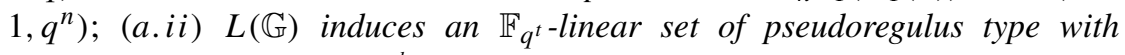
transversal points $\mathcal{I}$ and $\mathcal{I}^{\Upsilon_{1}^{l}}$. 
(b) If $t \geq 2$, then $L(\mathbb{G})$ is a scattered $\mathbb{F}_{q}$-linear set of rank tn of pseudoregulus type of $\Lambda$ with $\mathcal{I}$ and $\mathcal{I}^{\Upsilon_{1}^{l}}$ as transversal spaces.

Proof (a) If $t=1$, then $\mathbb{C}$ is an $\mathbb{F}_{q}$-subspace of $\mathbb{E}=\operatorname{End}\left(\mathbb{F}_{q^{n}}, \mathbb{F}_{q}\right)$; i.e. $L(\mathbb{G})$ is just an $(n-1)$-dimensional subspace of $\mathbb{P}$ contained in $\Lambda$. Note that the map

$$
\Phi:\left\{\left\langle t_{\lambda} \circ \varphi\right\rangle_{q}: \lambda \in \mathbb{F}_{q^{n}}^{*}\right\} \in \mathcal{D}_{1}(\mathcal{I}) \mapsto\left\langle\left(a_{0}, a_{1}, \ldots, a_{n-1}\right)\right\rangle_{q^{n}} \in P G\left(n-1, q^{n}\right),
$$

where $\varphi(x)=\sum_{i=0}^{n-1} a_{i} x^{q^{i}}$ is a linear collineation between $\Pi_{n-1}\left(\mathcal{D}_{1}(\mathcal{I})\right)$ and $P G(n-$ $\left.1, q^{n}\right)$ such that $\mathcal{I}^{\Phi}=\langle(1,0, \ldots, 0)\rangle_{q^{n}}, \mathcal{I}^{\Upsilon_{1}^{l} \Phi}=\langle(0, \ldots, 1, \ldots, 0)\rangle_{q^{n}}$ and $\Lambda^{\Phi}$ is the line of $P G\left(n-1, q^{n}\right)$ with equations $x_{i}=0$ for $i \neq 0, l$. Also $L(\mathbb{G})^{\Phi}=$ $\left\{\left\langle\left(y, 0,0, \ldots,-c y^{q^{m}}, 0, \ldots, 0\right)\right\rangle_{q^{n}}: y \in \mathbb{F}_{q^{n}}^{*}\right\}$ is an $\mathbb{F}_{q}$-linear set contained in the line $\Lambda^{\Phi}$ of $P G\left(n-1, q^{n}\right)$. By Definition $4.1, L(\mathbb{G})^{\Phi}$ is a maximum scattered $\mathbb{F}_{q}$-linear set of pseudoregulus type of $\Lambda^{\Phi}$, with transversal points $\mathcal{I}^{\Phi}$ and $\mathcal{I}^{\Upsilon_{1}^{l} \Phi}$. This proves (a).

(b) If $t \geq 2$, then the collineation

$$
\Phi_{f}:\left\langle t_{y}: x \mapsto y x\right\rangle_{q^{t}} \in \mathcal{I} \mapsto\left\langle f\left(t_{y}\right): x \mapsto-c y^{q^{m}} x^{q^{l t}}\right\rangle_{q^{t}} \in \mathcal{I}^{\Upsilon_{1}^{l}},
$$

is a semilinear collineation between $\mathcal{I}$ and $\mathcal{I}^{\Upsilon_{1}^{l}}$ with companion automorphism $\sigma: \alpha \in \mathbb{F}_{q^{t}} \mapsto \alpha^{q^{m}} \in \mathbb{F}_{q^{t}}$ and, since $\operatorname{gcd}(t, m)=1, \operatorname{Fix}(\sigma)=\mathbb{F}_{q}$. Hence, by Theorem 3.5, $L_{W_{1, f}}$ is an $\mathbb{F}_{q}$-linear set of $\Lambda$ of pseudoregulus type with transversal spaces $\mathcal{I}$ and $\mathcal{I}^{\Upsilon_{1}^{l}}$, and since

$$
W_{1, f}=\left\{t_{y}+f\left(t_{y}\right): y \in \mathbb{F}_{q^{n t}}\right\}=\left\{x \mapsto y x-c y^{q^{m}} x^{q^{t l}} \mid y \in \mathbb{F}_{q^{n t}}\right\}=\mathbb{C} .
$$

Case (b) follows.

Now, we will prove that the properties of $L(\mathbb{G})$ described in Proposition 5.5 completely characterize, up to isotopy, the Generalized Twisted Fields.

Theorem 5.6 Let $\mathbb{S}$ be a presemifield of order $q^{\text {nt }}$ with $\mathbb{F}_{q}$ contained in its center and $\mathbb{F}_{q^{t}}$ contained in its left nucleus and let $L(\mathbb{S})$ be the associated linear set with respect to the pair $\left(\mathbb{F}_{q}, \mathbb{F}_{q^{t}}\right)$. Also, assume that $L(\mathbb{S})$ is contained in a $(2 n-1)$-dimensional subspace of $\mathbb{P}=P G\left(n^{2}-1, q^{t}\right)$ joining two conjugated $\mathcal{D}$-spaces $X$ and $X^{\prime}$ of $\mathbb{P}$. Suppose that either Case (a) or Case (b) below holds:

(a) $t=1$ and $L(\mathbb{S})$ induces, in the linear representation $\Pi_{n-1}\left(\mathcal{D}_{1}(X)\right) \simeq P G(n-$ $\left.1, q^{n}\right)$, an $\mathbb{F}_{q}$-linear set of pseudoregulus type of the line $P G\left(1, q^{n}\right)$ of $\Pi_{n-1}\left(\mathcal{D}_{1}(X)\right)$ joining the points $X$ and $X^{\prime}$, with transversal points $X$ and $X^{\prime}$;

(b) $t \geq 2$ and $L(\mathbb{S})$ is a maximum scattered $\mathbb{F}_{q}$-linear set of pseudoregulus type of $\left\langle X, X^{\prime}\right\rangle_{q^{t}}$ with $X$ and $X^{\prime}$ as transversal spaces;

then $\mathbb{S}$ is isotopic to a Generalized Twisted Field.

Proof Without loss of generality we may assume that $\mathbb{S}=\left(\mathbb{F}_{q^{n t}},+, *\right)$ with $\mathbb{F}_{q}$ contained in $\mathbb{K}$ and $\mathbb{F}_{q^{t}}$ contained in $\mathbb{N}_{l}$. Let $\mathbb{E}=\operatorname{End}\left(\mathbb{F}_{q^{n t}}, \mathbb{F}_{q}\right)$ and let $\mathbb{C}=\left\{\varphi_{y}: x \in\right.$ 
$\left.\mathbb{F}_{q^{n t}} \mapsto x * y \in \mathbb{F}_{q^{n t}} \mid y \in \mathbb{F}_{q^{n t}}\right\}$ be the spread set defined by $\mathbb{S}$. Note that describing $\mathbb{S}$ corresponds, up to isotopy, to describing the associated linear set $L(\mathbb{S})=L_{\mathbb{C}}$ in the projective space $\mathbb{P}=P G\left(\mathbb{E}, \mathbb{F}_{q^{t}}\right)=P G\left(n^{2}-1, q^{t}\right)$, up to the action of the group $H\left(\mathcal{S}_{n, n}\left(q^{t}\right)\right)$. Since all the $\mathcal{D}$-spaces of $\mathbb{P}$ belong to the same $H\left(\mathcal{S}_{n, n}\left(q^{t}\right)\right)$-orbit, we may assume, up to isotopy, that $X=\mathcal{I}$, so $X^{\prime}=\mathcal{I}^{\Upsilon_{1}^{l}}$ for some $l \in\{1, \ldots, n-1\}$ (see (19)).

(a) By Definition 4.1, $L(\mathbb{S})=L_{\rho, \tau}$ where $\rho \in \mathbb{F}_{q^{n}}^{*}$ and $\tau: x \mapsto x^{q^{m}}$ is an automorphism of $\mathbb{F}_{q^{n}}$ such that $\operatorname{gcd}(m, n)=1$. This implies that

$$
\mathbb{C}=\left\{\varphi_{y}: x \in \mathbb{F}_{q^{n}} \mapsto x y+\rho y^{q^{m}} x^{q^{l}} \in \mathbb{F}_{q^{n}} \mid y \in \mathbb{F}_{q^{n}}\right\} .
$$

Hence $x * y=x y-c y^{q^{m}} x^{q^{l}}$ where $c=-\rho$, i.e. $\mathbb{S}$, up to isotopy, is a Generalized Twisted Field.

(b) By Theorem 3.12, $L(\mathbb{S})$ is of type $L_{\rho, f}$ with transversal spaces $\mathcal{I}$ and $\mathcal{I}^{\Upsilon_{1}^{l}}$. Hence, there exist a semilinear collineation

$$
\Phi_{f}:\left\langle t_{y}\right\rangle_{q^{t}} \in \mathcal{I} \mapsto\left\langle f\left(t_{y}\right)\right\rangle_{q^{t}} \in \mathcal{I}^{\Upsilon_{1}^{l}}
$$

with companion automorphism $\sigma \in \operatorname{Aut}\left(\mathbb{F}_{q^{t}}\right)$ such that $\operatorname{Fix}(\sigma)=\mathbb{F}_{q}$ and an element $\rho \in \mathbb{F}_{q^{n t}}^{*}$ such that

$$
\mathbb{C}=\left\{t_{y}+\rho f\left(t_{y}\right): y \in \mathbb{F}_{q^{n t}}\right\} .
$$

This implies that

$$
f\left(t_{y}\right): x \mapsto \eta y^{q^{m}} x^{q^{t l}},
$$

where $\eta \in \mathbb{F}_{q^{n t}}^{*}, 1 \leq m \leq n t-1$ and $\operatorname{gcd}(t, m)=1$. Hence, putting $c=-\eta \rho$, we have

$$
\mathbb{C}=\left\{\varphi_{y}: x \in \mathbb{F}_{q^{n t}} \mapsto x y-c y^{q^{m}} x^{q^{t l}} \in \mathbb{F}_{q^{n t}} \mid y \in \mathbb{F}_{q^{n t}}\right\},
$$

this means that $x * y=x y-c y^{q^{m}} x^{q^{t l}}$, and hence $\mathbb{S}$, up to isotopy, is a Generalized Twisted Field.

Note that if $n=2$ then $t \geq 2$ and by Remark 5.4, we can restate Theorem 5.6 as follows, which is a generalization of [5, Theorems 4.3, 3.7] and [25, Theorems 4.12, 4.13].

Corollary 5.7 Let $\mathbb{S}$ be a presemifield of order $q^{2 t}$ with center $\mathbb{F}_{q}$ and left nucleus $\mathbb{F}_{q^{t}}$. If $L(\mathbb{S})$ is an $\mathbb{F}_{q}$-linear set of $P G\left(3, q^{t}\right)$ of pseudoregulus type with transversal lines external to the quadric $\mathcal{S}_{2,2}=Q^{+}\left(3, q^{t}\right)$ pairwise polar with respect to the polarity defined by $Q^{+}\left(3, q^{t}\right)$, then $\mathbb{S}$ is isotopic to a Generalized Twisted Field.

\subsection{2-Dimensional Knuth semifields}

The Knuth semifields 2-dimensional over the left nucleus and $2 t$-dimensional $(t \geq 2)$ over the center $\mathbb{F}_{q}$ are the following: 
$\mathbb{K}_{17}=\left(\mathbb{F}_{q^{t}} \times \mathbb{F}_{q^{t}},+, *\right)$ and $\mathbb{K}_{19}=\left(\mathbb{F}_{q^{t}} \times \mathbb{F}_{q^{t}},+, \star\right)$ (see [6, p. 241 (multiplications (17) and (19))]), with

$$
(u, v) *(x, y)=(u, v)\left(\begin{array}{cc}
x & y \\
f y^{\sigma} & x^{\sigma}+y^{\sigma} g
\end{array}\right)
$$

and

$$
(u, v) \star(x, y)=(u, v)\left(\begin{array}{cc}
x & y \\
f y^{\sigma^{-1}} & x^{\sigma}+y g
\end{array}\right),
$$

where $\sigma \in \operatorname{Aut}\left(\mathbb{F}_{q^{t}}\right)$, Fix $\sigma=\mathbb{F}_{q}$, and $f$ and $g$ are non-zero elements in $\mathbb{F}_{q^{t}}$ such that the polynomial $x^{q+1}+g x-f$ has no root in $\mathbb{F}_{q^{t}}$.

The spread sets (of matrices) associated with $\mathbb{K}_{17}$ and $\mathbb{K}_{19}$ are

$$
\mathbb{C}_{17}=\left\{\left(\begin{array}{cc}
x & y \\
f y^{\sigma} & x^{\sigma}+y^{\sigma} g
\end{array}\right): x, y \in \mathbb{F}_{q^{t}}\right\} \subset \mathbb{M}
$$

and

$$
\mathbb{C}_{19}=\left\{\left(\begin{array}{cc}
x & y \\
f y^{\sigma^{-1}} & x^{\sigma}+y g
\end{array}\right): x, y \in \mathbb{F}_{q^{t}}\right\} \subset \mathbb{M},
$$

respectively, where $\mathbb{M}=\mathbb{M}\left(2, q^{t}\right)$ is the vector space of the $2 \times 2$-matrices over $\mathbb{F}_{q^{t}}$.

The sets $\mathbb{C}_{17}$ and $\mathbb{C}_{19}$ are $\mathbb{F}_{q}$-subspaces of $\mathbb{M}$ of dimension $2 t$ and hence they define $\mathbb{F}_{q}$-linear sets of rank $2 t$ in the projective space $\mathbb{P}=P G\left(\mathbb{M}, \mathbb{F}_{q^{t}}\right)=P G\left(3, q^{t}\right)$. Precisely, using the coordinatization $\left(\begin{array}{ll}x_{0} & x_{1} \\ x_{2} & x_{3}\end{array}\right) \mapsto\left(x_{0}, x_{1}, x_{2}, x_{3}\right)$,

$$
L\left(\mathbb{K}_{17}\right)=\left\{\left\langle\left(x, y, f y^{\sigma}, x^{\sigma}+g y^{\sigma}\right)\right\rangle_{q^{t}}: x, y \in \mathbb{F}_{q^{t}},(x, y) \neq(0,0)\right\}
$$

and

$$
L\left(\mathbb{K}_{19}\right)=\left\{\left\langle\left(x, y, f y^{\sigma^{-1}}, x^{\sigma}+g y\right)\right\rangle_{q^{t}}: x, y \in \mathbb{F}_{q^{t}},(x, y) \neq(0,0)\right\}
$$

are the relevant linear sets associated with the semifields $\mathbb{K}_{17}$ and $\mathbb{K}_{19}$, respectively.

Recall that $L\left(\mathbb{K}_{17}\right)$ and $L\left(\mathbb{K}_{19}\right)$ are disjoint from the hyperbolic quadric $Q^{+}\left(3, q^{t}\right)$ of $\mathbb{P}$ defined by the non-invertible matrices of $\mathbb{M}$. Let $\mathcal{R}_{1}$ be the regulus of $Q^{+}\left(3, q^{t}\right)$ containing the line $x_{2}=x_{3}=0$ and let $\mathcal{R}_{2}$ be the opposite one.

Remark 5.8 Note that the collineation $\Phi_{T}$ of $\mathbb{P}$ defined by the transpose operation on matrices fixes the quadric $Q^{+}\left(3, q^{t}\right)$ and interchanges the reguli $\mathcal{R}_{1}$ and $\mathcal{R}_{2}$ and $\Phi_{T}\left(L\left(\mathbb{K}_{17}(\sigma, f, g)\right)\right)=L\left(\mathbb{K}_{19}\left(\sigma, \frac{1}{f^{-1}}, \frac{g}{f}\right)\right)$. In other words, the family $\mathbb{K}_{19}$ is the transpose family of $\mathbb{K}_{17}$ (see [12, Sect. 5]).

Proposition 5.9 (1) $L\left(\mathbb{K}_{17}\right)$ is an $\mathbb{F}_{q}$-linear set of $\mathbb{P}=P G\left(3, q^{t}\right)$ of pseudoregulus type, whose transversal lines belong to $\mathcal{R}_{1}$.

(2) $L\left(\mathbb{K}_{19}\right)$ is an $\mathbb{F}_{q}$-linear set of $\mathbb{P}=P G\left(3, q^{t}\right)$ of pseudoregulus type, whose transversal lines belong to $\mathcal{R}_{2}$. 
Proof Let $r$ and $r^{\prime}$ be the lines of $\mathcal{R}_{1}$ with equations $r: x_{2}=x_{3}=0$ and $r^{\prime}: x_{0}=$ $x_{1}=0$. Then the map

$$
f:(x, y, 0,0) \mapsto\left(0,0, f y^{\sigma}, x^{\sigma}+g y^{\sigma}\right)
$$

induces a semilinear collineation $\Phi_{f}$ between $r$ and $r^{\prime}$ having $\sigma$ as a companion automorphism. Then, by Theorem $3.5, L_{1, f}$ is an $\mathbb{F}_{q}$-linear set of pseudoregulus type. Since $L_{1, f}=L\left(\mathbb{K}_{17}\right)$ we get (1).

Case (2) follows from Remark 5.8 and Case (1).

In the next theorem we prove that the descriptions of $L\left(\mathbb{K}_{17}\right)$ and $L\left(\mathbb{K}_{19}\right)$ given in Proposition 5.9 characterize the semifields $\mathbb{K}_{17}$ and $\mathbb{K}_{19}$ up to isotopism, generalizing some results contained in [5] and [25] for $t=2$.

Theorem 5.10 Let $\mathbb{S}$ be a presemifield of order $q^{2 t}$ with $\mathbb{F}_{q}$ contained in its center and $\mathbb{F}_{q^{t}}$ contained in its left nucleus and let $L(\mathbb{S})$ be the associated linear set with respect to the pair $\left(\mathbb{F}_{q}, \mathbb{F}_{q^{t}}\right)$. If $L(\mathbb{S})$ is an $\mathbb{F}_{q}$-linear set of pseudoregulus type of $\mathbb{P}=P G\left(3, q^{t}\right)$ with associated transversal lines $r$ and $r^{\prime}$ contained in $Q^{+}\left(3, q^{t}\right)$, then $\mathbb{S}$ is isotopic to a Knuth semifield $\mathbb{K}_{17}$ or $\mathbb{K}_{19}$. Precisely, if $r, r^{\prime} \in \mathcal{R}_{1}$, then $\mathbb{S}$ is isotopic to a semifield $\mathbb{K}_{17}$, whereas, if $r, r^{\prime} \in \mathcal{R}_{2}$, then $\mathbb{S}$ is isotopic to a semifield $\mathbb{K}_{19}$.

Proof Without loss of generality, we may assume that $\mathbb{S}=\left(\mathbb{F}_{q^{t}} \times \mathbb{F}_{q^{t}},+, \circ\right)$, with $\mathbb{F}_{q^{t}} \times\{0\}$ contained in its left nucleus. This implies that

$$
(u, v) \circ(x, y)=(u, v) M,
$$

where $M=M_{x, y} \in \mathbb{M}$. So, the spread set of matrices associated with $\mathbb{S}$ is

$$
\mathbb{C}=\left\{M_{x, y}=\left(\begin{array}{ll}
m_{0}(x, y) & m_{1}(x, y) \\
m_{2}(x, y) & m_{3}(x, y)
\end{array}\right): x, y \in \mathbb{F}_{q^{t}}\right\}
$$

and

$$
\begin{aligned}
L_{\mathbb{C}} & =L(\mathbb{S}) \\
& =\left\{\left\langle\left(m_{0}(x, y), m_{1}(x, y), m_{2}(x, y), m_{3}(x, y)\right)\right\rangle_{q^{t}}: x, y \in \mathbb{F}_{q^{t}},(x, y) \neq(0,0)\right\},
\end{aligned}
$$

where $m_{i}(x, y)$ are $\mathbb{F}_{q}$-linear maps. Assume that the transversal lines $r$ and $r^{\prime}$ of $L(\mathbb{S})$ are contained in $\mathcal{R}_{1}$. Since the group $H\left(\mathcal{S}_{2,2}\right)=G$ (see Remark 5.4) acts 2-transitively on the lines of $\mathcal{R}_{1}$, we can suppose that $r=\left\{\left\langle\left(x_{0}, x_{1}, 0,0\right)\right\rangle_{q^{t}}: x_{0}, x_{1} \in\right.$ $\left.\mathbb{F}_{q^{t}},(x, y) \neq(0,0)\right\}$ and $r^{\prime}=\left\{\left\langle\left(0,0, x_{2}, x_{3}\right)\right\rangle_{q^{t}}: x_{2}, x_{3} \in \mathbb{F}_{q^{t}},(x, y) \neq(0,0)\right\}$. Note that the stabilizer $G_{\left\{r, r^{\prime}\right\}}$ in the group $G$ of the lines $r$ and $r^{\prime}$ acts transitively on the points of $r$. If $P$ is any point of $r$, then the stabilizer $G_{\left\{r, r^{\prime}, P\right\}}$ of $P$ in $G_{\left\{r, r^{\prime}\right\}}$ fixes the point $P^{\perp} \cap r^{\prime}$ and acts transitively on the remaining points of $r^{\prime}$. This means that we can suppose, without loss of generality, that the line $s$ with equations $x_{1}=x_{2}=0$ belongs to the pseudoregulus associated with $L(\mathbb{S})$. Let $R=r \cap s=\langle(1,0,0,0)\rangle_{q^{t}}$ and $R^{\prime}=r^{\prime} \cap s=\langle(0,0,0,1)\rangle_{q^{t}}$. By Theorems 3.13 and 3.5 there exist a semilinear collineation $\Phi:\langle(x, y, 0,0)\rangle_{q^{t}} \in r \mapsto\langle(0,0, h(x, y), g(x, y))\rangle_{q^{t}} \in r^{\prime}$ having 
$\sigma \in \operatorname{Aut}\left(\mathbb{F}_{q^{t}}\right)$, with Fix $\sigma=\mathbb{F}_{q}$, as companion automorphism, and an element $\rho \in \mathbb{F}_{q^{t}}^{*}$ such that

$$
L(\mathbb{S})=\left\{\langle(x, y, \rho h(x, y), \rho g(x, y))\rangle_{q^{t}}: x, y \in \mathbb{F}_{q^{t}},(x, y) \neq(0,0)\right\} .
$$

Since $\Phi$ is semilinear with companion automorphism $\sigma$, we have that

$$
h(x, y)=a_{1} x^{\sigma}+a_{2} y^{\sigma} \quad \text { and } \quad g(x, y)=b_{1} x^{\sigma}+b_{2} y^{\sigma},
$$

where $a_{1}, a_{2}, b_{1}, b_{2} \in \mathbb{F}_{q^{t}}$. Also, since the line $s$ belongs to the pseudoregulus associated with $L(\mathbb{S})$, we have $\Phi(R)=R^{\prime}$, i.e. $h(1,0)=a_{1}=0$. So $L(\mathbb{S})=$ $\left\{\left\langle\left(x, y, \alpha y^{\sigma}, \beta x^{\sigma}+\gamma y^{\sigma}\right)\right\rangle_{q^{t}}: x, y \in \mathbb{F}_{q^{t}},(x, y) \neq(0,0)\right\}$, where $\alpha=\rho a_{2}, \beta=\rho b_{1}$, $\gamma=\rho b_{2}$. Note that $L(\mathbb{S}) \cap Q^{+}\left(3, q^{t}\right)=\emptyset$ implies $\beta \neq 0$. The collineation $\omega$ of $\mathbb{P}$ defined as $\omega:\left\langle\left(x_{0}, x_{1}, x_{2}, x_{3}\right)\right\rangle_{q^{t}} \mapsto\left\langle\left(x_{0}, x_{1}, x_{2} / \beta, x_{3} / \beta\right)\right\rangle_{q^{t}}$ fixes the reguli of $Q^{+}\left(3, q^{t}\right)$ and

$$
L(\mathbb{S})^{\omega}=\left\{\left\langle\left.\left(x, y, f y^{\sigma}, x^{\sigma}+g y^{\sigma}\right)\right|_{q^{t}}: x, y \in \mathbb{F}_{q^{t}},(x, y) \neq(0,0)\right\},\right.
$$

where $f=\frac{\alpha}{\beta}$ and $g=\frac{\gamma}{\beta}$, i.e., up to isotopy, $\mathbb{S}$ is a $\mathbb{K}_{17}$ Knuth semifield. Finally, if the transversal lines of $L(\mathbb{S})$ belong to $\mathcal{R}_{2}$, arguing as in the previous case, we get that $\mathbb{S}$ is isotopic to a $\mathbb{K}_{19}$ Knuth semifield.

Acknowledgements We thank the referees for their valuable comments; these have increased the readability of the article. Also, we wish to thank G. Donati and N. Durante for their helpful suggestions regarding Remark 4.2.

\section{References}

1. Albert, A.A.: Isotopy for generalized twisted fields. An. Acad. Bras. Ciênc. 33, 265-275 (1961)

2. Albert, A.A.: Generalized twisted fields. Pac. J. Math. 11, 1-8 (1961)

3. Bader, L., Lunardon, G.: Desarguesian spreads. Ric. Mat. 60, 15-37 (2011)

4. Blokhuis, A., Lavraw, M.: Scattered spaces with respect to a spread in $P G(n, q)$. Geom. Dedic. 81(13), 231-243 (2000)

5. Cardinali, I., Polverino, O., Trombetti, R.: Semifield planes of order $q^{4}$ with kernel $\mathbb{F}_{q^{2}}$ and center $\mathbb{F}_{q}$. Eur. J. Comb. 27, 940-961 (2006)

6. Dembowski, P.: Finite Geometries. Springer, Berlin (1968)

7. Donati, G., Durante, N.: Scattered linear sets generated by collineations between pencils of lines, submitted

8. Freeman, J.W.: Reguli and pseudo-reguli in $P G\left(3, s^{2}\right)$. Geom. Dedic. 9, 267-280 (1980)

9. Harris, J.: Algebraic Geometry. A First Course. Springer, New York (1992)

10. Herzer, A.: Generalized Segre varieties. Rend. Mat. 7, 1-36 (1986)

11. Hirschfeld, J.W.P., Thas, J.A.: General Galois Geometries. Oxford University Press, Oxford (1991)

12. Knuth, D.E.: Finite semifields and projective planes. J. Algebra 2, 182-217 (1965)

13. Lavrauw, M.: On the isotopism classes of finite semifields. Finite Fields Appl. 14, 897-910 (2008)

14. Lavrauw, M.: Finite semifields with a large nucleus and higher secant varieties to Segre varieties. Adv. Geom. 11(3), 399-410 (2011)

15. Lavrauw, M., Marino, G., Polverino, O., Trombetti, R.: $\mathbb{F}_{q}$-pseudoreguli of $P G\left(3, q^{3}\right)$ and scattered semifields of order $q^{6}$. Finite Fields Appl. 17, 225-239 (2011)

16. Lavrauw, M., Polverino, O.: Finite semifields. In: De Beule, J., Storme, L. (eds.) Current Research Topics in Galois Geometry. NOVA Academic Publ., New York (2011) Chap. 6. ISBN: 978-1-61209523-3 
17. Lavrauw, M., Van de Voorde, G.: On linear sets on a projective line. Des. Codes Cryptogr. 56, 89-104 (2010)

18. Lavrauw, M., Van de Voorde, G.: Scattered linear sets and pseudoreguli. Electronic J. Comb. 20(1) (2013)

19. Lidl, R., Niederreiter, H.: Finite Fields. Encyclopedia Math. Appl., vol. 20. Addison-Wesley, Reading (1983). Now distributed by Cambridge University Press

20. Lunardon, G.: Normal spreads. Geom. Dedic. 75, 245-261 (1999)

21. Lunardon, G.: Translation ovoids. J. Geom. 76, 200-215 (2003)

22. Lunardon, G., Polverino, O.: Blocking sets and derivable partial spreads. J. Algebr. Comb. 14, 49-56 (2001)

23. Lunardon, G., Polverino, O.: Translation ovoids of orthogonal polar spaces. Forum Math. 16, 663-669 (2004)

24. Marino, G., Polverino, O.: On the nuclei of a finite semifield. In: Theory and Applications of Finite Fields. Contemp. Math., vol. 579, pp. 123-141. Amer. Math. Soc., Providence (2012)

25. Marino, G., Polverino, O., Trombetti, R.: On $\mathbb{F}_{q}$-linear sets of $P G\left(3, q^{3}\right)$ and semifields. J. Comb. Theory, Ser. A 114, 769-788 (2007)

26. Polverino, O.: Linear sets in finite projective spaces. Discrete Math. 310, 3096-3107 (2010)

27. Segre, B.: Teoria di Galois, fibrazioni proiettive e geometrie non desarguesiane. Ann. Mat. Pura Appl. 64, 1-76 (1964) 